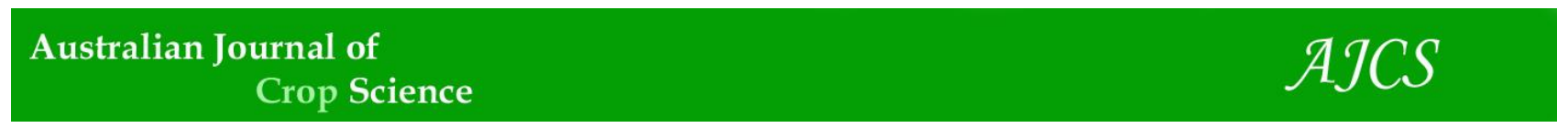

AJCS 14(07):1109-1120 (2020)

ISSN:1835-2707

doi: 10.21475/ajcs.20.14.07.p2277

\title{
Phenotypic response of barley landrace and its wild progenitor Hordeum spontaneum from Jordan to salt stress
}

\author{
Adel H. Abdel-Ghani ${ }^{1}$, Khalid Al-Abbsi ${ }^{1}$, Nael Thaher ${ }^{2 *}$, Saddam Al-Dalain ${ }^{3}$, Ghaid Al-Rabadi ${ }^{4}$, Farah Al- \\ Nasir $^{1}$
}

${ }^{1}$ Department of Plant Production, Faculty of Agriculture, Mutah University, Jordan

${ }^{2}$ National Agricultural Research Centre, Amman, Jordan

${ }^{3}$ Al-Shoubak University College, Al-Balqa Applied University, Jordan

${ }^{4}$ Department of Animal Production, Faculty of Agriculture, Mutah University, Jordan

*Corresponding author: n1thaher@gmail.com

Abstract

Populations of barley landrace [Hordeum vulgare (L.)] and wild barley progenitor [Hordeum spontaneum (L.)] were collected from 13 diverse eco-geographical regions in Jordan. In addition to two check varieties (Rum and Acsad 165) were collected from farmers' fields. In total, 28 genotypes were evaluated during germination, early seedling and maturity stages for salt tolerance. Barley populations were exposed to tap water of $0.85 \mathrm{dS} \mathrm{m}^{-1}$ and saline solutions $\left(\mathrm{NaCl}, \mathrm{CaCl}_{2}\right.$ and $\left.\mathrm{MgSO}_{4}\right)$ of $4,8,12$ and $16 \mathrm{dS} \mathrm{m}^{-1}$. Final germination percentage, the lengths of shoot and seminal root, growth and yield related traits were significantly decreased by increasing the level of salinity. Proline content was increased by increasing the level of salinity. A high level of salinity of $16 \mathrm{dS} \mathrm{m}{ }^{-1}$ had a strong impact on reduction of grain yield and other agronomic traits in the examined populations. A considerable variation among populations was detectable. Barley landrace and wild barley that were collected from Tafila region were the most tolerant grown populations in Jordan. Interestingly, the ranking pattern of these two populations at the germination and seedling relatedtraits was consistent with the ranking pattern at the maturity phase. In conclusion, this study indicates that barley landrace and wild barley populations are potential donors for genes of salt tolerance that can be used to enhance salinity tolerance in barley.

Keywords: barley landrace, germination, grain yield, proline, salt stress, semi-arid conditions, wild barley, yield components. Abbreviations: ANOVA_analysis of variance; Ca_calcium; $\mathrm{CaCl}_{2}$ calcium chloride; $\mathrm{Cl}$ _chloride; $\mathrm{cm}$ _centimeter; ${ }^{\circ} \mathrm{C}$ _degree centigrade; $\mathrm{dS} \mathrm{m}^{-1}$ _deci siemens per meter; $\mathrm{h}^{2}$ _heritability estimate; h_hour; K_potassium; kg_kilogram; mm_millimeter; $\mathrm{Mg} \_$magnesium; $\mathrm{MgSO}_{4}$ magnesium sulfate; $\mathrm{Na}$ _sodium; $\mathrm{NaCl}$ _sodium chloride; $\mathrm{NaOCl}$ _sodium hypochlorite; $\mathrm{O}_{2}$ dimer oxygen; S_sulfur; $\mathrm{SO}_{4}$ sulfate; SAS_statistical analysis software; SSR_simple sequence repeats; SNP_single nucleotide polymorphism; WI_value of recorded trait under salt stress; SI_value of recorded trait under non-salt stress; YD_mean of a particular trait in salt treatment; YP_mean value under non-stress treatment.

Introduction

Barley [Hordeum vulgare (L.)] is a major economic and important crop in the world. It has been used as a model crop for genetic and physiological studies (Koornneef et al., 1997; Takahashi et al., 2001; Abdel-Ghani et al., 2014). Barley is cultivated on mountains with an average of 300 $\mathrm{mm}$ rainfall and in desert fringes with an average of $100 \mathrm{~mm}$ rainfall in Jordan (Harlan, 1976; Abdel-Ghani et al., 2004). Barley landraces were developed and resulted from natural and farmer-direct selection of thousands of years (Harlan, 1975; Frankel et al., 1995; Abdel-Ghani et al., 2004). However, wild barley $H$. vulgare spp. spontaneum $(H$. spontaneum) was considered the progenitor of cultivated barley $H$. vulgare L spp. vulgare ( $H$. vulgare) (Zohary and Hopf, 2000). Barley landrace and its wild progenitor are both considered potential sources of useful genes for biotic and abiotic stresses. They are habitants of high rainfall regions as well as adapted to dry regions (Harlan, 1975, 1976; Blum et al., 1989; Jaradat et al., 2004a), offering a high value, drought-tolerant crop that increases diversity in the annual cropping system of this region. Salinity is a major problem that affects the economic yield of agricultural crops worldwide. High concentrations of salt cause deleterious effects on plant (Bernstein, 1962; Kramer and Boyer, 1996). Moreover, the intensive use of fertilizers and irrigation of saline water have resulted in salinization of agricultural soils, and therefore have dramatically led to the decrease of germination, plant growth and yield losses (Bernstein, 1962). Barley is the most salt-tolerant cereal crop compared to wheat crop (Maas, 1986; Forster et al., 1990). However, a significant level of genetic variability of barley population exists in their sensitivity to salt stress (Omara and Hussein, 1987; Troech and Thompson, 1993; Abdel-Ghani, 2009).

Salt-tolerant crops continue to perform under salt stress and have the potential to maintain a reasonable yield under such conditions (Zhu, 2001; Parida and Das, 2005). The response of plant to salt stress is a complex trait with a polygenic inheritance (Sousa et al., 2003; Carillo et al., 2012; Deinlien et al., 2014; Gupta and Huang, 2014). Plant breeders can 
directly select for grain yield or perform selection for traits related to salt stress (Nevo and Chen, 2010; Roy et al., 2014). The salt stress intensity index is used as an indicator on the performance of a genotype under salt stress comparable to non-stress conditions where tolerant genotypes display a minimum loss in grain yield (Sinha, 1987; Adjel et al., 2013; Allel et al., 2019). Various desirable traits such as high germination percentage and vigorous seedling are used for indirect selection to improve the adaptability of barley crop under salt stress (Jaradat et al., 2004b; Okcu et al., 2005; Zhang et al., 2010). In addition, other desirable traits are also considered in selection for salt tolerance such as the minimum levels of leaf injury, low $\mathrm{Na}^{+}$ or $\mathrm{Cl}^{-}$accumulation in leaves (Rahnama et al., 2011) and high $\mathrm{K}+\mathrm{Na}+$ ion ratio in plant tissue (Flowers and Hajibagheri, 2001; Chen et al., 2007; Shabala et al., 2007). Detection of barley genotypes to salt tolerance could allow these genotypes to be hybridized with elite barley genotypes in order to enhance their adaptability to salt stress. Furthermore, these genotypes could be used directly in the case of barley landrace for the same purpose. The objectives of this study were to: i) screen potential gene donors for salt tolerance in a set of barley genotypes (landrace and wild), ii) assess the response of barley landrace and its wild progenitor to the increased level of salinity at germination, seedling and maturity stages, and iii) estimate the variance component, heritability and genetic correlation coefficients.

\section{Results and discussion}

\section{Effect of salt stress on germination}

There were differences in response for salt stress within wild barley genotypes. The highest germination percentage at different levels of salt stress was obtained in the control treatment of $0.85 \mathrm{dS} \mathrm{m}^{-1}$. This was significantly higher than the final germination under salinization treatments of 8,12 and $16 \mathrm{dS} \mathrm{m}^{-1}$. However, the overall response of germination to low salinization treatment of $4 \mathrm{dS} \mathrm{m}^{-1}$ was not significantly different from the control. The responses of germination were averaged over genotypes based on the final germination and seedling attributes as shown in Table 1. The reduction in germination in barley landraces and two improved varieties was from 0.89 to $6.13 \%$ when the salinity was increased from 0.85 to $16 \mathrm{dS} \mathrm{m}^{-1}$. However, the reduction in the final germination in the wild genotype was $3.89 \%$ in salt-free medium to $28.90 \%$ at $16 \mathrm{dS} \mathrm{m}^{-1}$ (Table 2). The final germination percentage for landraces and two improved varieties exceeded 89 and $84 \%$ germination at 12 and $16 \mathrm{dS} \mathrm{m}^{-1}$, respectively (Table 3 ).

\section{Effect of salt stress on seedling growth}

Seedling, seminal and root length were drastically reduced in response to salt stress. The number of seminal roots and coleoptile length was the least affected seedling; whereas shoot and root dry weight were moderately reduced in response to salinity stress. Seminal root length was severely inhibited by salinity levels more than the shoots at low of 4 and $8 \mathrm{dS} \mathrm{m}^{-1}$ levels of salinity. The low salinity treatment of 4 dS $\mathrm{m}^{-1}$ had a significant effect in the reduction of seedling growth as compared to other treatments of 8,12 and $16 \mathrm{dS}$ $\mathrm{m}^{-1}$. However, wild genotypes had severe reduction in all seedling related traits as compared to barley landraces at treatment of $8 \mathrm{dS} \mathrm{m}^{-1}$ and higher (Tables 2 and 5).

\section{Effect of salt treatment on growth and yield traits}

There was significant effect in the reduction of plant above ground biomass (grain yield, biological and straw yield) and yield components (number of tillers per plant, number of grains per spike and thousand-kernel weight) for all salinity treatments (Table 4). The reduction was higher under high levels of salinity as compared with low levels of salinity. All agronomic traits displayed a significant genotype $\times$ salt treatment, except grain yield, number of fertile tillers and days to heading. In general, all over genotypes, the reduction in yield occurred due to the reduction in yield components (Tables 5 and 6). A few barley landraces and wild genotypes exhibited the lowest reduction in yield components at increased level of salt.

\section{Effect of salt stress on plant height and days to heading and maturity}

The increased level of salinity had significant decrease on the plant height. There were a variety and treatment interaction, which had a significant effect on plant height and phenological traits.

\section{Accumulation of proline in leaves}

Free proline accumulation in barley leaves was increased by increasing the severity of salinization. There was a significant interaction between variety and salt level on accumulation of proline in leaves (Table 4). Nevertheless, there was no association between content of proline in leaves and grain yield.

\section{Susceptibility index at different levels of salinity}

Stress susceptibility index 'S' for germination, seedling related-traits and agronomic parameters are presented in Tables 2, 3, 5 and 6 . The values of stress susceptibility index were increased by increasing the level of salinity for all recorded variables.

\section{Genetic variance and heritability estimates}

Genotype $\times$ salinity interactions were significant for final germination percentage, seedling length, seminal root length, seedling length, plant height, harvest index, phenological traits and content of proline. Heritability estimates $\left(h^{2}\right)$ ranged from 48.03 for days to heading to 0.98 for germination percentage. The variation was obvious among genotypes due to genotype, genotype $\times$ salinity level and phenotypic variances for final germination percentage, seedling related-traits and various morphological, agronomic, and phenological parameters as shown in Tables 7 and 8 . The results show that wild barley and cultivated barley are cross compatible, which indicate the possibility of introgression of cultivated barley with genes from wild barley gene pool. Therefore, incorporating genes from barley landrace and its wild relative may substantially improve the performance of barley varieties to tolerance of salt stress. Previous studies (Nevo et al., 1984; Weltzien, 1989; Weltzien and Fischbeck, 1990; Jaradat, 1991; Morrell and Clegg, 2007) showed that natural populations of $H$. spontaneum and barley landraces from the Fertile Crescent were major sources of genes for disease resistance and 
Table 1. Effect of different levels of salinity on some seedling characteristics of the 28 barley genotypes.

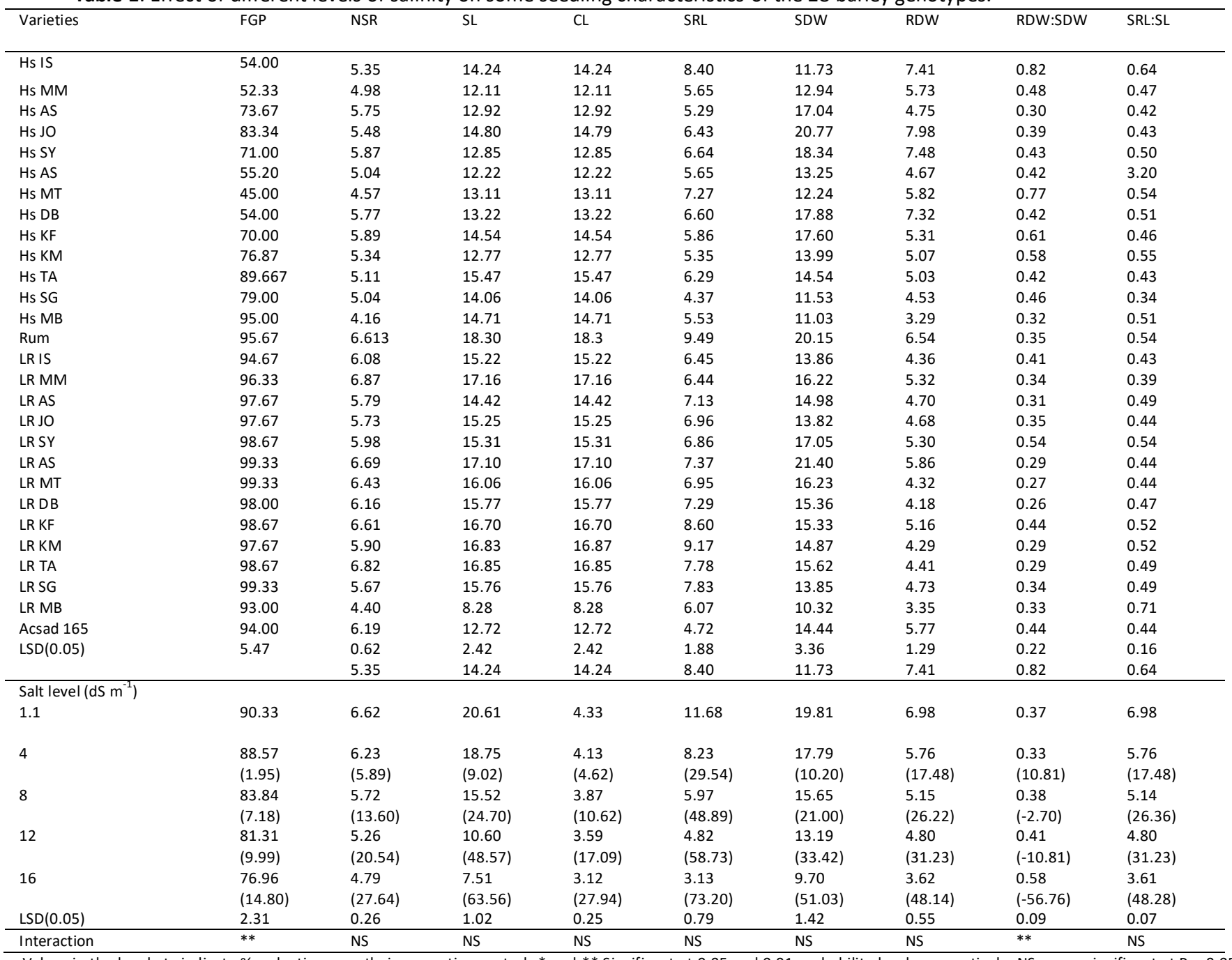

Values in the brackets indicate $\%$ reduction over their respective control. ${ }^{*}$ and ${ }^{* *}$ Significant at 0.05 and 0.01 probability levels, respectively. NS $=$ non-significant at $\mathrm{P}=0.05$ FGP, final germination percentage; NSR, number of seminal roots; SL, seedling length; CL, coleoptile length; SRL, seminal root length, SDW, shoot dry weight; RDW, root dry weight.

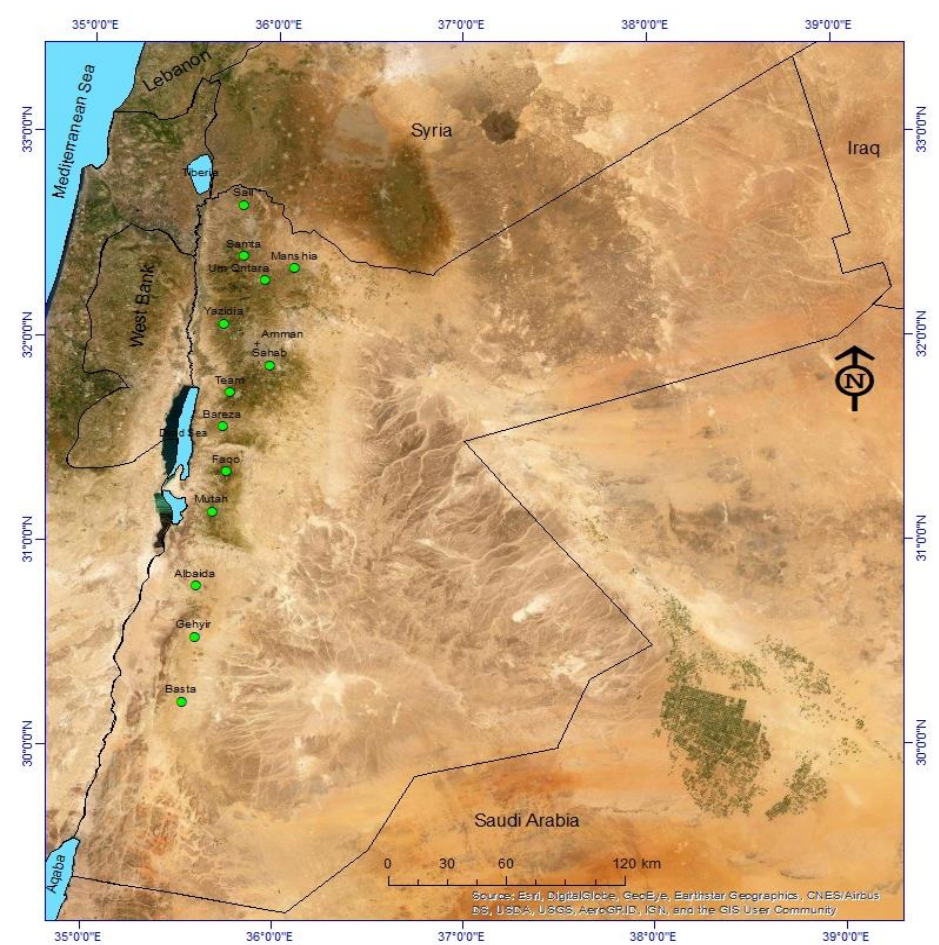

Fig 1. A map highlighting the collection sites of barley landrace and wild barley progenitor from 13 different sites, Jordan. 
Table 2. Salt susceptibility index $(S)$ and percent of reduction (PR) in seedling characteristics of 13 genotypes of $H$. spontaneum

\begin{tabular}{|c|c|c|c|c|c|c|c|c|c|c|c|c|c|c|c|}
\hline \multirow{2}{*}{$\begin{array}{l}\text { Salinity } \\
\text { level } \\
\left(\mathrm{dS} \mathrm{m} \mathrm{m}^{-1}\right)\end{array}$} & \multicolumn{8}{|c|}{$\mathrm{S}$} & \multicolumn{7}{|l|}{ PR } \\
\hline & Genotype & FGP & NSR & SL & $\mathrm{RL}$ & $\mathrm{CL}$ & SDW & RDW & FGP & NSR & SL & $\mathrm{RL}$ & $\mathrm{CL}$ & SDW & RDW \\
\hline \multirow[t]{16}{*}{4} & $\mathrm{Hs}$ IS & 0.00 & 0.16 & 0.36 & 1.98 & 0.43 & 0.59 & 0.12 & 0.00 & 0.96 & 3.28 & 58.56 & 2.00 & 6.04 & 2.04 \\
\hline & Hs MM & 1.35 & 2.70 & -0.07 & 1.27 & 0.63 & 0.19 & 0.97 & 2.63 & 15.90 & -0.65 & 37.64 & 2.92 & 1.94 & 17.00 \\
\hline & Hs AS & 1.01 & 1.89 & 0.40 & 0.50 & 1.28 & 0.38 & 0.15 & 1.96 & 11.11 & 3.62 & 14.65 & 5.93 & 3.84 & 2.61 \\
\hline & Hs JO & 0.95 & 1.92 & 1.90 & 1.47 & 1.39 & -0.05 & 0.27 & 1.85 & 11.30 & 17.13 & 43.39 & 6.42 & -0.49 & 4.69 \\
\hline & Hs SY & 1.01 & 0.48 & 0.93 & 1.58 & 0.18 & 0.25 & 1.92 & 1.96 & 2.80 & 8.41 & 46.74 & 0.82 & 2.59 & 33.51 \\
\hline & Hs AS & 2.18 & 0.50 & 0.41 & 0.91 & 2.11 & 2.76 & 1.61 & 4.26 & 2.94 & 3.68 & 26.99 & 9.76 & 28.15 & 28.13 \\
\hline & Hs MT & 15.02 & -0.21 & 0.50 & 0.72 & 1.06 & 0.43 & 0.08 & 29.27 & -1.27 & 4.55 & 21.26 & 4.88 & 4.36 & 1.35 \\
\hline & Hs DB & 1.47 & 1.37 & 1.55 & 1.22 & 0.72 & 0.37 & 2.71 & 2.86 & 8.08 & 13.95 & 35.98 & 3.33 & 3.73 & 47.34 \\
\hline & $\mathrm{Hs} \mathrm{KF}$ & 1.07 & 0.67 & 1.03 & 1.47 & 0.36 & 0.96 & 0.70 & 2.08 & 3.96 & 9.29 & 43.32 & 1.68 & 9.74 & 12.17 \\
\hline & Hs KM & 1.01 & 0.00 & 2.29 & 1.12 & 1.05 & 1.86 & 0.96 & 1.96 & 0.00 & 20.69 & 33.09 & 4.84 & 18.99 & 16.73 \\
\hline & Hs TA & 1.80 & 0.55 & 0.13 & 1.40 & 0.65 & 0.12 & 2.18 & 3.51 & 3.23 & 1.21 & 41.50 & 3.01 & 1.19 & 38.12 \\
\hline & Hs SG & -0.90 & 0.99 & 2.24 & 1.88 & 0.39 & 1.12 & 1.05 & -1.75 & 5.81 & 20.19 & 55.41 & 1.80 & 11.41 & 18.34 \\
\hline & $\mathrm{Hs} \mathrm{MB}$ & 0.00 & 0.25 & 0.03 & 0.41 & 3.31 & 0.48 & 0.50 & 0.00 & 1.45 & 0.31 & 12.11 & 15.30 & 4.86 & 8.74 \\
\hline & Min. & -0.90 & -0.21 & -0.07 & 0.41 & 0.18 & -0.05 & 0.08 & -1.75 & -1.27 & -0.65 & 12.11 & 0.82 & -0.49 & 1.35 \\
\hline & Max. & 15.02 & 2.70 & 2.29 & 1.98 & 3.31 & 2.76 & 2.71 & 29.27 & 15.90 & 20.69 & 58.56 & 15.30 & 28.15 & 47.34 \\
\hline & Mean & 2.00 & 0.87 & 0.90 & 1.23 & 1.04 & 0.73 & 1.02 & 3.89 & 5.10 & 8.13 & 36.20 & 4.82 & 7.41 & 17.75 \\
\hline \multirow[t]{16}{*}{8} & $\mathrm{Hs}$ IS & 1.25 & 6.12 & 2.89 & 1.90 & 2.36 & 2.23 & 0.32 & 2.44 & 36.06 & 26.12 & 56.17 & 10.89 & 22.76 & 5.56 \\
\hline & $\mathrm{Hs} \mathrm{MM}$ & 20.26 & 2.65 & 4.44 & 2.15 & 2.38 & 0.48 & 1.55 & 39.47 & 15.63 & 40.11 & 63.60 & 11.01 & 4.92 & 27.07 \\
\hline & Hs AS & 7.04 & 1.54 & 3.43 & 1.62 & 1.79 & 0.86 & 0.56 & 13.73 & 9.09 & 30.98 & 47.74 & 8.26 & 8.74 & 9.80 \\
\hline & Hs JO & 4.75 & 3.92 & 3.15 & 2.08 & -0.60 & 1.18 & 0.28 & 9.26 & 23.08 & 28.44 & 61.38 & -2.75 & 12.03 & 4.86 \\
\hline & Hs SY & 12.08 & 3.93 & 4.51 & 2.50 & 0.89 & 3.41 & 2.11 & 23.53 & 23.13 & 40.68 & 73.84 & 4.10 & 34.79 & 36.81 \\
\hline & Hs AS & 3.55 & 3.74 & 3.59 & 1.85 & 2.73 & 3.81 & 1.61 & 6.91 & 22.06 & 32.40 & 54.60 & 12.60 & 38.86 & 28.17 \\
\hline & Hs MT & 18.78 & 1.61 & 2.23 & 2.09 & 3.34 & 2.74 & 1.23 & 36.59 & 9.49 & 20.10 & 61.71 & 15.45 & 27.91 & 21.43 \\
\hline & Hs DB & 2.93 & 1.83 & 4.19 & 1.56 & 0.90 & 1.33 & 2.91 & 5.71 & 10.77 & 37.84 & 46.07 & 4.17 & 13.56 & 50.89 \\
\hline & $\mathrm{Hs} \mathrm{KF}$ & 9.62 & 2.86 & 2.75 & 1.76 & 0.18 & 1.17 & 0.53 & 18.75 & 16.83 & 24.82 & 51.93 & 0.84 & 11.97 & 9.24 \\
\hline & Hs KM & 4.43 & 2.53 & 3.87 & 1.34 & 3.84 & 2.39 & 1.35 & 8.63 & 14.89 & 34.89 & 39.58 & 17.74 & 24.38 & 23.59 \\
\hline & Hs TA & 3.60 & 2.01 & 2.50 & 1.96 & 3.09 & 0.31 & 2.44 & 7.02 & 11.83 & 22.52 & 57.75 & 14.29 & 3.16 & 42.56 \\
\hline & Hs SG & 13.51 & 1.38 & 3.05 & 2.01 & 1.76 & 1.53 & 2.80 & 26.32 & 8.14 & 27.54 & 59.40 & 8.11 & 15.63 & 48.98 \\
\hline & Hs MB & 0.00 & 1.23 & 2.20 & 1.05 & 8.28 & 2.98 & 1.08 & 0.00 & 7.25 & 19.84 & 31.14 & 38.25 & 30.44 & 18.88 \\
\hline & Min. & 0.00 & 1.23 & 2.20 & 1.05 & -0.60 & 0.31 & 0.28 & 0.00 & 7.25 & 19.84 & 31.14 & -2.75 & 3.16 & 4.86 \\
\hline & Max. & 20.26 & 6.12 & 4.51 & 2.50 & 8.28 & 3.81 & 2.91 & 39.47 & 36.06 & 40.68 & 73.84 & 38.25 & 38.86 & 50.89 \\
\hline & Mean & 7.83 & 2.72 & 3.29 & 1.84 & 2.38 & 1.88 & 1.44 & 15.26 & 16.02 & 29.71 & 54.22 & 11.00 & 19.16 & 25.22 \\
\hline \multirow[t]{16}{*}{12} & Hs IS & 13.77 & 6.69 & 8.10 & 2.43 & 8.19 & 5.49 & -3.47 & 26.83 & 39.42 & 73.13 & 71.82 & 37.83 & 56.01 & -60.71 \\
\hline & Hs MM & 8.10 & 3.98 & 8.40 & 2.68 & 4.96 & 4.72 & 1.34 & 15.79 & 23.45 & 75.83 & 79.21 & 22.92 & 48.15 & 23.36 \\
\hline & Hs AS & 14.09 & 3.86 & 6.66 & 1.91 & 2.24 & 2.12 & 1.00 & 27.45 & 22.73 & 60.11 & 56.45 & 10.34 & 21.60 & 17.52 \\
\hline & Hs JO & 5.70 & 6.37 & 5.59 & 2.11 & 1.79 & 2.95 & 3.52 & 11.11 & 37.50 & 50.46 & 62.43 & 8.26 & 30.05 & 61.57 \\
\hline & Hs SY & 16.10 & 5.71 & 5.63 & 2.28 & 0.71 & 3.67 & 2.08 & 31.37 & 33.64 & 50.85 & 67.44 & 3.28 & 37.42 & 36.38 \\
\hline & Hs AS & 7.64 & 5.77 & 6.49 & 2.13 & 3.02 & 3.22 & 1.90 & 14.89 & 33.99 & 58.58 & 62.88 & 13.96 & 32.83 & 33.27 \\
\hline & Hs MT & 22.53 & 4.19 & 7.13 & 2.56 & 5.63 & 4.14 & -0.17 & 43.90 & 24.68 & 64.34 & 75.48 & 26.02 & 42.25 & -2.89 \\
\hline & Hs DB & 5.87 & 3.11 & 6.61 & 2.04 & 1.71 & 3.93 & 2.82 & 11.43 & 18.35 & 59.62 & 60.33 & 7.92 & 40.03 & 49.37 \\
\hline & $\mathrm{Hs} \mathrm{KF}$ & 9.62 & 3.99 & 7.15 & 2.19 & 4.14 & 1.63 & 0.67 & 18.75 & 23.51 & 64.55 & 64.69 & 19.12 & 16.64 & 11.64 \\
\hline & Hs KM & 6.04 & 4.74 & 7.93 & 1.92 & 7.16 & 4.72 & 2.68 & 11.76 & 27.93 & 71.60 & 56.80 & 33.06 & 48.09 & 46.76 \\
\hline & Hs TA & 4.50 & 4.75 & 4.01 & 1.77 & 4.72 & 2.93 & 1.62 & 8.77 & 27.96 & 36.16 & 52.33 & 21.80 & 29.92 & 28.27 \\
\hline & Hs SG & 14.41 & 2.71 & 5.89 & 2.11 & 1.76 & 2.61 & 3.03 & 28.07 & 15.99 & 53.13 & 62.31 & 8.11 & 26.64 & 52.91 \\
\hline & $\mathrm{Hs} \mathrm{MB}$ & 0.88 & 3.20 & 6.27 & 1.98 & 9.57 & 3.42 & 1.59 & 1.72 & 18.84 & 56.58 & 58.48 & 44.21 & 34.92 & 27.83 \\
\hline & Min & 0.88 & 2.71 & 4.01 & 1.77 & 0.71 & 1.63 & -3.47 & 1.72 & 15.99 & 36.16 & 52.33 & 3.28 & 16.64 & -60.71 \\
\hline & Max. & 22.53 & 6.69 & 8.40 & 2.68 & 9.57 & 5.49 & 3.52 & 43.90 & 39.42 & 75.83 & 79.21 & 44.21 & 56.01 & 61.57 \\
\hline & Mean & 9.94 & 4.54 & 6.61 & 2.16 & 4.28 & 3.50 & 1.43 & 19.37 & 26.77 & 59.61 & 63.90 & 19.76 & 35.73 & 25.02 \\
\hline \multirow[t]{16}{*}{16} & Hs IS & 38.81 & 6.37 & 8.72 & 2.75 & 12.27 & 7.54 & 1.36 & 75.61 & 37.50 & 78.73 & 81.28 & 56.67 & 76.92 & 23.81 \\
\hline & $\mathrm{Hs} \mathrm{MM}$ & 14.86 & 7.21 & 7.93 & 2.75 & 7.38 & 2.54 & 1.48 & 28.95 & 42.50 & 71.56 & 81.27 & 34.08 & 25.85 & 25.89 \\
\hline & Hs AS & 12.08 & 3.66 & 6.84 & 2.27 & 2.87 & 3.26 & 1.44 & 23.53 & 21.55 & 61.70 & 67.14 & 13.28 & 33.21 & 25.13 \\
\hline & Hs JO & 7.60 & 5.55 & 7.17 & 2.63 & 2.18 & 5.80 & 3.66 & 14.81 & 32.69 & 64.74 & 77.82 & 10.09 & 59.18 & 64.01 \\
\hline & Hs SY & 13.08 & 4.92 & 8.13 & 2.72 & 6.98 & 5.97 & 3.61 & 25.49 & 28.97 & 73.39 & 80.23 & 32.24 & 60.88 & 63.11 \\
\hline & Hs AS & 17.20 & 11.98 & 9.33 & 3.23 & 11.09 & 6.88 & 4.06 & 33.51 & 70.59 & 84.24 & 95.40 & 51.22 & 70.12 & 70.89 \\
\hline & Hs MT & 31.30 & 5.69 & 7.45 & 2.65 & 15.05 & 8.45 & 1.20 & 60.98 & 33.54 & 67.22 & 78.26 & 69.51 & 86.14 & 21.05 \\
\hline & Hs DB & 8.80 & 4.33 & 6.70 & 2.35 & 2.71 & 4.35 & 4.25 & 17.14 & 25.51 & 60.49 & 69.45 & 12.50 & 44.31 & 74.29 \\
\hline & Hs KF & 11.76 & 3.11 & 9.39 & 2.68 & 7.78 & 8.55 & 0.45 & 22.92 & 18.32 & 84.76 & 79.23 & 35.92 & 87.23 & 7.85 \\
\hline & Hs KM & 13.08 & 5.24 & 9.24 & 2.56 & 9.60 & 6.32 & 3.16 & 25.49 & 30.85 & 83.38 & 75.49 & 44.35 & 64.47 & 55.15 \\
\hline & Hs TA & 4.50 & 7.57 & 7.73 & 2.59 & 7.65 & 4.88 & 2.12 & 8.77 & 44.62 & 69.72 & 76.44 & 35.34 & 49.77 & 37.09 \\
\hline & Hs SG & 16.21 & 5.13 & 7.66 & 2.58 & 4.10 & 6.30 & 3.45 & 31.58 & 30.23 & 69.17 & 76.22 & 18.92 & 64.22 & 60.25 \\
\hline & Hs MB & 3.54 & 3.44 & 8.71 & 2.00 & 11.12 & 5.86 & 1.79 & 6.90 & 20.29 & 78.59 & 59.17 & 51.37 & 59.76 & 31.23 \\
\hline & Min. & 3.54 & 3.11 & 6.70 & 2.00 & 2.18 & 2.54 & 0.45 & 6.90 & 18.32 & 60.49 & 59.17 & 10.09 & 25.85 & 7.85 \\
\hline & Max. & 38.81 & 11.98 & 9.39 & 3.23 & 15.05 & 8.55 & 4.25 & 75.61 & 70.59 & 84.76 & 95.40 & 69.51 & 87.23 & 74.29 \\
\hline & Mean & 14.83 & 5.71 & 8.08 & 2.60 & 7.75 & 5.90 & 2.46 & 28.90 & 33.63 & 72.90 & 76.72 & 35.81 & 60.16 & 43.06 \\
\hline
\end{tabular}

FGP, final germination percentage; NSR, number of seminal roots; SL, seedling length; CL, coleoptile length; SRL, seminal root length, SDW, shoot dry weight; RDW, root dry weight. 
Table 3. Salt susceptibility index (S) and percent of reduction (PR) in seedling related-traits of 13 genotypes of landrace and two improved varieties of barley.

\begin{tabular}{|c|c|c|c|c|c|c|c|c|c|c|c|c|c|c|c|}
\hline \multirow{2}{*}{$\begin{array}{c}\text { Salinity } \\
\text { level } \\
\left(\mathrm{dS} \mathrm{m}^{-1}\right)\end{array}$} & \multicolumn{8}{|c|}{$S$} & \multicolumn{7}{|c|}{$P R$} \\
\hline & Genotype & FGP & NSR & SL & $\mathrm{RL}$ & $\mathrm{CL}$ & SDW & RDW & FGP & NSR & SL & RL & $\mathrm{CL}$ & SDW & RDW \\
\hline \multirow[t]{18}{*}{4} & Rum & 0.86 & 1.48 & 0.82 & 0.31 & 0.76 & 1.38 & 0.12 & 1.67 & 8.70 & 7.39 & 9.10 & 3.51 & 14.03 & 2.06 \\
\hline & LR IS & 0.86 & 0.24 & 0.16 & 0.89 & 1.11 & 3.04 & 1.17 & 1.67 & 1.42 & 1.40 & 26.32 & 5.13 & 30.97 & 20.49 \\
\hline & LR MM & 0.86 & 0.87 & 1.63 & 0.26 & 0.50 & 0.28 & 0.60 & 1.67 & 5.13 & 14.67 & 7.64 & 2.33 & 2.81 & 10.55 \\
\hline & LR AS & 0.00 & 0.18 & 0.71 & 0.97 & -0.16 & 1.81 & 1.89 & 0.00 & 1.05 & 6.38 & 28.68 & -0.74 & 18.43 & 33.04 \\
\hline & LR JO & 0.86 & 1.07 & 1.66 & 0.29 & 0.23 & 0.84 & 0.96 & 1.67 & 6.32 & 14.95 & 8.68 & 1.07 & 8.52 & 16.71 \\
\hline & LR SY & 0.00 & 1.04 & 0.45 & 0.23 & 1.57 & 0.47 & 0.94 & 0.00 & 6.12 & 4.11 & 6.79 & 7.26 & 4.76 & 16.43 \\
\hline & LR AS & 0.86 & 0.46 & 1.12 & 0.45 & 0.83 & 0.68 & 1.11 & 1.67 & 2.70 & 10.10 & 13.29 & 3.85 & 6.94 & 19.43 \\
\hline & LR MT & 0.00 & 2.57 & 1.39 & 0.00 & -0.18 & 1.00 & 0.38 & 0.00 & 15.14 & 12.54 & 0.00 & -0.83 & 10.22 & 6.67 \\
\hline & LR DB & 0.00 & 2.00 & 2.16 & 0.37 & 1.31 & 0.54 & 0.63 & 0.00 & 11.76 & 19.53 & 10.82 & 6.06 & 5.48 & 11.03 \\
\hline & LR KF & 0.00 & 1.08 & 0.94 & 1.51 & 0.00 & 2.78 & 0.16 & 0.00 & 6.36 & 8.45 & 44.64 & 0.00 & 28.38 & 2.78 \\
\hline & LR KM & 0.00 & 1.37 & 1.59 & 0.91 & 1.62 & 1.62 & 0.28 & 0.00 & 8.08 & 14.36 & 26.92 & 7.46 & 16.50 & 4.81 \\
\hline & LR TA & 0.00 & 1.05 & 1.01 & 0.41 & 2.89 & 0.91 & -1.30 & 0.00 & 6.19 & 9.08 & 12.00 & 13.34 & 9.29 & -22.68 \\
\hline & LR SG & 0.00 & 0.37 & -0.15 & 0.61 & 1.51 & 0.89 & 1.99 & 0.00 & 2.20 & -1.39 & 17.94 & 6.98 & 9.03 & 34.76 \\
\hline & LR MB & 0.87 & 1.51 & 1.65 & 2.00 & 1.73 & 0.88 & 2.20 & 1.69 & 8.92 & 14.92 & 58.97 & 8.00 & 8.95 & 38.41 \\
\hline & Acsad 165 & 1.74 & 0.63 & 0.83 & 1.64 & -0.18 & 1.18 & 0.23 & 3.39 & 3.70 & 7.49 & 48.39 & -0.81 & 12.04 & 3.97 \\
\hline & Min & 0.00 & 0.18 & -0.15 & 0.00 & -0.18 & 0.28 & -1.30 & 0.00 & 1.05 & -1.39 & 0.00 & -0.83 & 2.81 & -22.68 \\
\hline & Max & 1.74 & 2.57 & 2.16 & 2.00 & 2.89 & 3.04 & 2.20 & 3.39 & 15.14 & 19.53 & 58.97 & 13.34 & 30.97 & 38.41 \\
\hline & Mean & 0.46 & 1.06 & 1.06 & 0.72 & 0.90 & 1.22 & 0.76 & 0.89 & 6.25 & 9.60 & 21.35 & 4.17 & 12.42 & 13.23 \\
\hline 8 & Rum & 1.71 & 3.25 & 2.69 & 1.25 & 4.62 & 1.57 & 0.82 & 3.33 & 19.13 & 24.27 & 36.92 & 21.34 & 15.97 & 14.33 \\
\hline & LR IS & 2.57 & 2.82 & 1.61 & 1.61 & 3.33 & 4.22 & 2.47 & 5.00 & 16.59 & 14.49 & 47.70 & 15.38 & 43.08 & 43.12 \\
\hline & LR MM & 0.86 & 2.61 & 1.82 & 1.17 & 2.01 & 0.70 & 0.94 & 1.67 & 15.38 & 16.47 & 34.55 & 9.30 & 7.17 & 16.35 \\
\hline & LR AS & 0.86 & 1.61 & 3.25 & 1.98 & 1.11 & 2.47 & 2.39 & 1.67 & 9.47 & 29.30 & 58.60 & 5.15 & 25.22 & 41.78 \\
\hline & LR JO & 0.86 & 1.07 & 1.84 & 1.01 & 0.89 & 2.47 & 1.76 & 1.67 & 6.32 & 16.61 & 29.93 & 4.11 & 25.14 & 30.82 \\
\hline & LR SY & 0.86 & 0.91 & 1.55 & 0.62 & 2.49 & 1.21 & 1.41 & 1.67 & 5.36 & 13.96 & 18.40 & 11.50 & 12.34 & 24.71 \\
\hline & LR AS & 0.00 & 0.76 & 3.01 & 1.33 & 2.32 & 2.74 & 1.23 & 0.00 & 4.50 & 27.13 & 39.27 & 10.71 & 27.99 & 21.45 \\
\hline & LR MT & 0.00 & 1.71 & 2.57 & 1.25 & -1.80 & 1.79 & 1.05 & 0.00 & 10.09 & 23.22 & 36.82 & -8.33 & 18.28 & 18.29 \\
\hline & LR DB & 0.00 & 1.66 & 2.43 & 0.82 & 1.31 & 3.38 & 2.16 & 0.00 & 9.80 & 21.89 & 24.26 & 6.06 & 34.42 & 37.68 \\
\hline & LR KF & 0.86 & 1.39 & 2.64 & 1.41 & 1.15 & 3.69 & 0.47 & 1.67 & 8.18 & 23.78 & 41.58 & 5.33 & 37.66 & 8.23 \\
\hline & LR KM & 0.86 & 1.54 & 2.00 & 1.27 & 2.10 & 1.91 & 1.26 & 1.67 & 9.09 & 18.03 & 37.39 & 9.70 & 19.43 & 22.02 \\
\hline & LR TA & 0.86 & 1.35 & 1.41 & 1.86 & 3.19 & 1.25 & -0.11 & 1.67 & 7.96 & 12.74 & 54.93 & 14.72 & 12.72 & -1.89 \\
\hline & LR SG & 0.00 & 1.12 & 1.99 & 1.86 & 1.45 & 1.42 & 2.33 & 0.00 & 6.59 & 17.94 & 55.03 & 6.72 & 14.48 & 40.64 \\
\hline & LR MB & 1.74 & 4.02 & 2.80 & 1.98 & 3.18 & 1.62 & 2.36 & 3.39 & 23.69 & 25.31 & 58.41 & 14.67 & 16.53 & 41.33 \\
\hline & Acsad 165 & 2.61 & 2.51 & 2.55 & 2.16 & 1.20 & 1.72 & 0.58 & 5.08 & 14.81 & 23.01 & 63.80 & 5.55 & 17.50 & 10.15 \\
\hline & Min. & 0.00 & 0.76 & 1.41 & 0.62 & -1.80 & 0.70 & -0.11 & 0.00 & 4.50 & 12.74 & 18.40 & -8.33 & 7.17 & -1.89 \\
\hline & Max. & 2.61 & 4.02 & 3.25 & 2.16 & 4.62 & 4.22 & 2.47 & 5.08 & 23.69 & 29.30 & 63.80 & 21.34 & 43.08 & 43.12 \\
\hline & Mean & 0.97 & 1.89 & 2.28 & 1.44 & 1.90 & 2.14 & 1.41 & 1.90 & 11.13 & 20.54 & 42.51 & 8.79 & 21.86 & 24.60 \\
\hline 12 & Rum & 4.28 & 3.39 & 4.67 & 1.44 & 4.88 & 2.29 & 1.41 & 8.33 & 20.00 & 42.12 & 42.55 & 22.56 & 23.36 & 24.66 \\
\hline & LR IS & 4.28 & 3.46 & 3.90 & 1.47 & 2.33 & 4.51 & 2.37 & 8.33 & 20.38 & 35.20 & 43.50 & 10.77 & 45.95 & 41.37 \\
\hline & LR MM & 3.42 & 3.19 & 4.50 & 1.68 & 3.19 & 2.62 & 1.78 & 6.67 & 18.80 & 40.57 & 49.64 & 14.73 & 26.73 & 31.13 \\
\hline & LR AS & 0.86 & 1.97 & 5.36 & 2.33 & 1.75 & 2.54 & 3.04 & 1.67 & 11.58 & 48.34 & 68.83 & 8.09 & 25.92 & 53.14 \\
\hline & LR JO & 0.86 & 2.14 & 3.46 & 1.70 & 4.60 & 2.96 & 2.01 & 1.67 & 12.63 & 31.23 & 50.33 & 21.23 & 30.19 & 35.18 \\
\hline & LR SY & 0.86 & 1.91 & 4.50 & 1.20 & 2.99 & 2.94 & 2.69 & 1.67 & 11.22 & 40.60 & 35.47 & 13.81 & 30.01 & 47.06 \\
\hline & LR AS & 0.00 & 1.99 & 4.63 & 1.64 & 3.51 & 3.93 & 2.30 & 0.00 & 11.71 & 41.79 & 48.34 & 16.21 & 40.04 & 40.14 \\
\hline & LR MT & 0.00 & 2.08 & 3.38 & 1.54 & -2.96 & 2.84 & 1.32 & 0.00 & 12.23 & 30.55 & 48.34 & 16.21 & 28.99 & 40.14 \\
\hline & LR D B & 2.57 & 2.00 & 4.26 & 1.20 & 3.93 & 3.49 & 2.38 & 5.00 & 11.76 & 38.46 & 35.41 & 18.14 & 35.64 & 41.56 \\
\hline & LR KF & 0.86 & 2.47 & 4.55 & 1.76 & 2.54 & 3.84 & 1.38 & 1.67 & 14.55 & 41.05 & 51.91 & 11.73 & 39.18 & 24.04 \\
\hline & LR KM & 2.57 & 2.91 & 5.53 & 2.20 & 2.54 & 2.95 & 1.50 & 5.00 & 17.17 & 49.93 & 64.87 & 11.75 & 30.09 & 26.30 \\
\hline & LR TA & 0.86 & 2.25 & 4.48 & 1.95 & 4.08 & 2.24 & -1.02 & 1.67 & 13.27 & 40.45 & 57.73 & 18.84 & 22.80 & -17.89 \\
\hline & LR SG & 0.00 & 1.87 & 3.58 & 2.21 & 2.01 & 1.16 & 2.38 & 0.00 & 10.99 & 32.29 & 65.41 & 9.30 & 11.82 & 41.61 \\
\hline & LR MB & 4.35 & 4.86 & 5.15 & 2.12 & 6.64 & 4.12 & 3.06 & 8.47 & 28.62 & 46.50 & 62.64 & 30.67 & 42.05 & 53.47 \\
\hline & Acsad 165 & 3.48 & 2.62 & 2.28 & 2.22 & 2.33 & 3.86 & 0.86 & 6.78 & 15.43 & 20.57 & 65.47 & 10.77 & 39.38 & 15.05 \\
\hline & Min. & 0.00 & 1.87 & 2.28 & 1.20 & -2.96 & 1.16 & -1.02 & 0.00 & 10.99 & 20.57 & 35.41 & -13.67 & 11.82 & -17.89 \\
\hline & Max. & 4.35 & 4.86 & 5.53 & 2.33 & 6.64 & 4.51 & 3.06 & 8.47 & 28.62 & 49.93 & 68.83 & 30.67 & 45.95 & 53.47 \\
\hline & Mean & 1.95 & 2.61 & 4.28 & 1.78 & 2.96 & 3.09 & 1.83 & 3.79 & 15.36 & 38.64 & 52.51 & 13.66 & 31.48 & 31.99 \\
\hline 16 & Rum & 4.28 & 3.54 & 4.81 & 2.26 & 6.07 & 2.70 & 2.02 & 8.33 & 20.87 & 43.38 & 66.83 & 28.05 & 27.53 & 35.38 \\
\hline & LR IS & 5.99 & 4.99 & 5.44 & 2.17 & 2.50 & 4.88 & 3.02 & 11.67 & 29.38 & 49.12 & 64.14 & 11.54 & 49.74 & 52.71 \\
\hline & LR MM & 4.28 & 3.48 & 4.76 & 1.93 & 3.19 & 3.28 & 2.52 & 8.33 & 20.51 & 42.96 & 57.09 & 14.73 & 33.48 & 44.01 \\
\hline & LR AS & 4.28 & 3.57 & 6.87 & 2.62 & 2.71 & 3.14 & 3.26 & 8.33 & 21.05 & 62.03 & 77.31 & 12.50 & 32.03 & 56.94 \\
\hline & LR JO & 3.42 & 3.75 & 6.34 & 2.30 & 4.75 & 4.32 & 2.45 & 6.67 & 22.11 & 57.23 & 67.85 & 21.92 & 44.02 & 42.82 \\
\hline & LR SY & 1.71 & 3.29 & 7.11 & 1.74 & 3.72 & 4.42 & 2.83 & 3.33 & 19.39 & 64.20 & 51.32 & 17.17 & 45.08 & 49.50 \\
\hline & LR AS & 0.86 & 4.94 & 6.97 & 2.21 & 8.06 & 5.42 & 3.11 & 1.67 & 29.13 & 62.91 & 65.26 & 37.23 & 55.32 & 54.36 \\
\hline & LR MT & 1.71 & 3.43 & 6.74 & 2.22 & 1.53 & 6.39 & 3.05 & 3.33 & 20.18 & 60.84 & 65.71 & 7.08 & 65.14 & 53.33 \\
\hline & LR DB & 2.57 & 2.33 & 7.79 & 2.40 & 6.85 & 4.71 & 4.83 & 5.00 & 13.73 & 70.27 & 70.82 & 31.65 & 48.06 & 84.42 \\
\hline & LR KF & 1.71 & 3.39 & 7.51 & 2.70 & 5.20 & 4.70 & 1.79 & 3.33 & 20.00 & 67.77 & 79.65 & 24.00 & 47.96 & 31.32 \\
\hline & LR KM & 2.57 & 3.26 & 6.16 & 2.60 & 3.23 & 3.32 & 2.71 & 5.00 & 19.19 & 55.60 & 76.85 & 14.93 & 33.82 & 47.41 \\
\hline & LR TA & 1.71 & 3.35 & 3.91 & 2.17 & 4.58 & 2.97 & 2.33 & 3.33 & 19.76 & 35.24 & 64.00 & 21.14 & 30.25 & 40.80 \\
\hline & LR SG & 1.71 & 2.18 & 4.35 & 2.26 & 2.13 & 3.12 & 2.51 & 3.33 & 12.82 & 39.26 & 66.68 & 9.82 & 31.78 & 43.79 \\
\hline & LR MB & 6.96 & 5.48 & 6.10 & 2.92 & 6.98 & 4.32 & 3.04 & 13.56 & 32.31 & 55.06 & 86.32 & 32.22 & 44.06 & 53.16 \\
\hline & Acsad 165 & 3.48 & 6.13 & 6.70 & 2.32 & 3.76 & 4.26 & 1.89 & 6.78 & 36.11 & 60.49 & 68.64 & 17.35 & 43.42 & 32.98 \\
\hline & Min. & 0.86 & 2.18 & 3.91 & 1.74 & 1.53 & 2.70 & 1.79 & 1.67 & 12.82 & 35.24 & 51.32 & 7.08 & 27.53 & 31.32 \\
\hline & Max. & 6.96 & 6.13 & 7.79 & 2.92 & 8.06 & 6.39 & 4.83 & 13.56 & 36.11 & 70.27 & 86.32 & 37.23 & 65.14 & 84.42 \\
\hline & Mean & 3.15 & 3.81 & 6.10 & 2.32 & 4.35 & 4.13 & 2.76 & 6.13 & 22.44 & 55.09 & 68.56 & 20.09 & 42.11 & 48.20 \\
\hline
\end{tabular}


Table 4. Effect of different levels of salinity on agronomic parameters and proline accumulation in leaves of the 28 genotypes of barley.

\begin{tabular}{|c|c|c|c|c|c|c|c|c|c|c|c|c|}
\hline $\begin{array}{l}\text { Genotype } \\
\mathrm{s}\end{array}$ & $\mathrm{PH}$ & GY & BY & SY & $\mathrm{HI}$ & NT & HSW & SS & $\overline{\mathrm{DH}}$ & $\mathrm{DM}$ & GFP & Proline \\
\hline $\mathrm{Hs}$ IS & 90.80 & 2.07 & 6.82 & 4.38 & 0.32 & 5.26 & 3.33 & 11.15 & 95.35 & 135.35 & 39.90 & 9.14 \\
\hline Hs MM & 84.33 & 1.58 & 6.63 & 4.27 & 0.24 & 4.68 & 3.31 & 10.28 & 92.55 & 134.55 & 42.00 & 8.95 \\
\hline Hs AS & 82.93 & 1.66 & 6.56 & 4.77 & 0.27 & 4.31 & 3.57 & 11.06 & 94.90 & 135.35 & 40.45 & 7.66 \\
\hline Hs JO & 80.33 & 1.91 & 7.06 & 4.85 & 0.27 & 4.60 & 3.28 & 12.46 & 92.70 & 132.30 & 39.60 & 8.86 \\
\hline $\mathrm{HsSY}$ & 83.63 & 2.06 & 7.74 & 5.50 & 0.27 & 5.06 & 3.56 & 11.28 & 96.50 & 134.95 & 38.45 & 9.25 \\
\hline Hs AS & 92.35 & 2.12 & 7.36 & 5.15 & 0.28 & 5.15 & 3.19 & 12.95 & 98.70 & 131.95 & 33.25 & 11.31 \\
\hline $\mathrm{Hs}$ MT & 93.30 & 1.47 & 6.14 & 4.39 & 0.23 & 4.35 & 3.03 & 11.21 & 93.45 & 134.70 & 41.25 & 9.11 \\
\hline $\mathrm{Hs} D \mathrm{DB}$ & 86.38 & 1.66 & 6.43 & 4.65 & 0.26 & 4.45 & 3.33 & 11.17 & 93.75 & 130.85 & 37.10 & 16.79 \\
\hline $\mathrm{Hs} \mathrm{KF}$ & 90.65 & 2.19 & 6.77 & 4.56 & 0.33 & 4.32 & 3.59 & 12.14 & 93.75 & 129.95 & 35.80 & 14.47 \\
\hline $\mathrm{Hs} \mathrm{KM}$ & 87.68 & 1.78 & 6.28 & 4.48 & 0.30 & 4.37 & 3.25 & 12.82 & 94.15 & 130.90 & 35.95 & 12.23 \\
\hline Hs TA & 89.10 & 2.31 & 6.11 & 4.08 & 0.38 & 5.67 & 3.35 & 12.19 & 92.60 & 132.65 & 40.05 & 22.16 \\
\hline $\mathrm{Hs} \mathrm{SG}$ & 82.20 & 1.64 & 7.25 & 4.82 & 0.22 & 5.08 & 2.75 & 11.54 & 92.75 & 131.45 & 38.45 & 18.91 \\
\hline $\mathrm{Hs} \mathrm{MB}$ & 82.12 & 1.93 & 6.18 & 4.59 & 0.29 & 4.79 & 3.22 & 12.01 & 92.65 & 132.75 & 40.10 & 14.58 \\
\hline Rum & 66.45 & 1.83 & 4.68 & 3.73 & 0.38 & 2.20 & 4.95 & 16.04 & 88.20 & 125.90 & 37.70 & 16.87 \\
\hline LR IS & 70.30 & 2.13 & 6.61 & 4.55 & 0.32 & 4.28 & 5.63 & 8.76 & 87.95 & 124.55 & 36.60 & 15.27 \\
\hline LR MM & 66.25 & 2.61 & 6.66 & 3.52 & 0.39 & 3.24 & 5.13 & 15.70 & 89.70 & 128.65 & 38.95 & 16.09 \\
\hline LR AS & 67.20 & 2.25 & 6.25 & 3.87 & 0.36 & 4.48 & 5.35 & 9.81 & 90.55 & 127.40 & 36.85 & 15.66 \\
\hline LR JO & 73.10 & 2.33 & 6.45 & 4.12 & 0.36 & 4.10 & 5.24 & 11.03 & 90.10 & 128.50 & 38.40 & 12.90 \\
\hline LR SY & 72.40 & 1.90 & 6.21 & 3.87 & 0.31 & 3.45 & 5.08 & 10.33 & 89.45 & 126.95 & 37.50 & 14.742 \\
\hline LR AS & 67.30 & 2.00 & 7.26 & 4.47 & 0.31 & 3.58 & 5.26 & 10.75 & 89.55 & 125.75 & 36.20 & 14.58 \\
\hline LR MT & 72.05 & 2.17 & 6.04 & 3.55 & 0.35 & 4.52 & 5.37 & 8.91 & 88.60 & 125.15 & 36.55 & 15.23 \\
\hline LR DB & 73.93 & 2.79 & 7.36 & 4.38 & 0.38 & 4.73 & 5.30 & 11.59 & 89.05 & 125.40 & 36.35 & 14.13 \\
\hline LR KF & 73.25 & 2.45 & 7.09 & 4.53 & 0.5 & 4.47 & 5.26 & 10.69 & 87.95 & 124.25 & 36.30 & 12.87 \\
\hline LR KM & 66.85 & 2.90 & 7.65 & 4.58 & 0.38 & 5.31 & 5.05 & 10.83 & 88.00 & 124.25 & 36.15 & 13.29 \\
\hline LR TA & 69.30 & 2.13 & 7.13 & 4.83 & 0.29 & 5.37 & 5.09 & 8.04 & 89.45 & 125.25 & 35.80 & 16.53 \\
\hline LR SG & 66.20 & 2.09 & 6.23 & 4.10 & 0.33 & 5.01 & 5.16 & 8.38 & 88.45 & 125.80 & 37.25 & 16.55 \\
\hline LR MB & 64.03 & 3.32 & 6.65 & 4.21 & 0.35 & 5.44 & 5.61 & 7.66 & 89.05 & 123.60 & 34.55 & 15.47 \\
\hline Acsad 165 & 65.50 & 1.01 & 6.53 & 5.18 & 0.14 & 3.67 & 4.73 & 5.57 & 87.00 & 122.60 & 35.60 & 20.74 \\
\hline $\operatorname{LSD}(0.05)$ & 7.42 & 0.41 & 0.99 & 0.72 & 0.09 & 0.75 & 0.45 & 1.73 & 3.04 & 2.03 & 3.62 & 3.86 \\
\hline \multicolumn{13}{|l|}{$\begin{array}{l}\begin{array}{l}\text { Salt level } \\
\left(\mathrm{dS} \mathrm{m}^{-1}\right)\end{array} \\
\end{array}$} \\
\hline 0.85 & 83.41 & 2.83 & 7.53 & 4.90 & 0.38 & 5.15 & 4.83 & 12.45 & 92.65 & 130.46 & 37.80 & 4.90 \\
\hline 4 & $\begin{array}{l}79.06 \\
(5.22)\end{array}$ & $\begin{array}{l}2.21 \\
(21.91)\end{array}$ & $\begin{array}{l}6.53 \\
(13.28)\end{array}$ & $\begin{array}{l}4.24 \\
(13.47)\end{array}$ & $\begin{array}{l}0.34 \\
(10.53)\end{array}$ & $\begin{array}{l}4.52 \\
(12.23)\end{array}$ & $\begin{array}{l}4.53 \\
(6.21)\end{array}$ & $\begin{array}{l}11.39 \\
(8.51)\end{array}$ & $\begin{array}{l}93.21 \\
(-0.60)\end{array}$ & $\begin{array}{l}132.08 \\
(-1.24)\end{array}$ & $\begin{array}{l}38.83 \\
(-2.72)\end{array}$ & $\begin{array}{l}7.34 \\
(-49.80)\end{array}$ \\
\hline 8 & 78.27 & 1.90 & 6.39 & 4.25 & 0.30 & 4.42 & 4.27 & 10.75 & 89.63 & 127.81 & 38.18 & 10.29 \\
\hline & (6.16) & (32.86) & (15.14) & (13.27) & (21.05) & (14.17) & (11.59) & (13.65) & (3.26) & (2.03) & $(-1.01)$ & $(-110.00)$ \\
\hline 12 & $\begin{array}{l}74.21 \\
(11.03)\end{array}$ & $\begin{array}{l}1.70 \\
(39.93)\end{array}$ & $\begin{array}{l}6.62 \\
(12.08)\end{array}$ & $\begin{array}{l}4.48 \\
(8.57)\end{array}$ & $\begin{array}{l}0.26 \\
(31.58)\end{array}$ & $\begin{array}{l}4.28 \\
(16.89)\end{array}$ & $\begin{array}{l}4.10 \\
(15.11)\end{array}$ & $\begin{array}{l}10.14 \\
(18.55)\end{array}$ & $\begin{array}{l}90.84 \\
(1.95)\end{array}$ & $\begin{array}{l}128.00 \\
(1.89)\end{array}$ & $\begin{array}{l}37.16 \\
(1.69)\end{array}$ & $\begin{array}{l}21.83 \\
(-345.51)\end{array}$ \\
\hline 16 & 70.86 & 1.60 & 6.15 & 4.06 & 0.27 & 4.29 & 3.88 & 9.75 & 90.64 & 126.56 & 36.10 & 26.06 \\
\hline ISD (0.05) & $\begin{array}{l}15.05 \\
(3.14)\end{array}$ & 43.46 & 18.33 & $\begin{array}{l}17.14 \\
(0.26)\end{array}$ & 28.95 & 16.70 & 19.67 & $\begin{array}{l}21.69 \\
(0.72)\end{array}$ & $\begin{array}{l}2.17 \\
(1.28)\end{array}$ & $\begin{array}{l}2.99 \\
(0.93)\end{array}$ & $\begin{array}{l}4.50 \\
(1.53)\end{array}$ & -431.84 \\
\hline $\begin{array}{l}\text { Interactio } \\
\mathrm{n}\end{array}$ & $* *$ & $* *$ & NS & NS & NS & $*$ & ** & ** & * & ** & & \\
\hline
\end{tabular}

Values in the brackets indicate \% reduction over their respective control.

$\mathrm{PH}$, plant height; GY, grain yield; BY, biological yield, SY, straw yield; HI, harvest index; NT, number of tillers; HSW, hundred seed weight; SS, seeds per spike; DH, days to heading; DM, days to mat urity; GFP, grain filling period.

Table 5. Salt susceptibility index (S) and percent of reduction (PR) in agronomic traits of 13 genotypes of $H$. spontaneum.

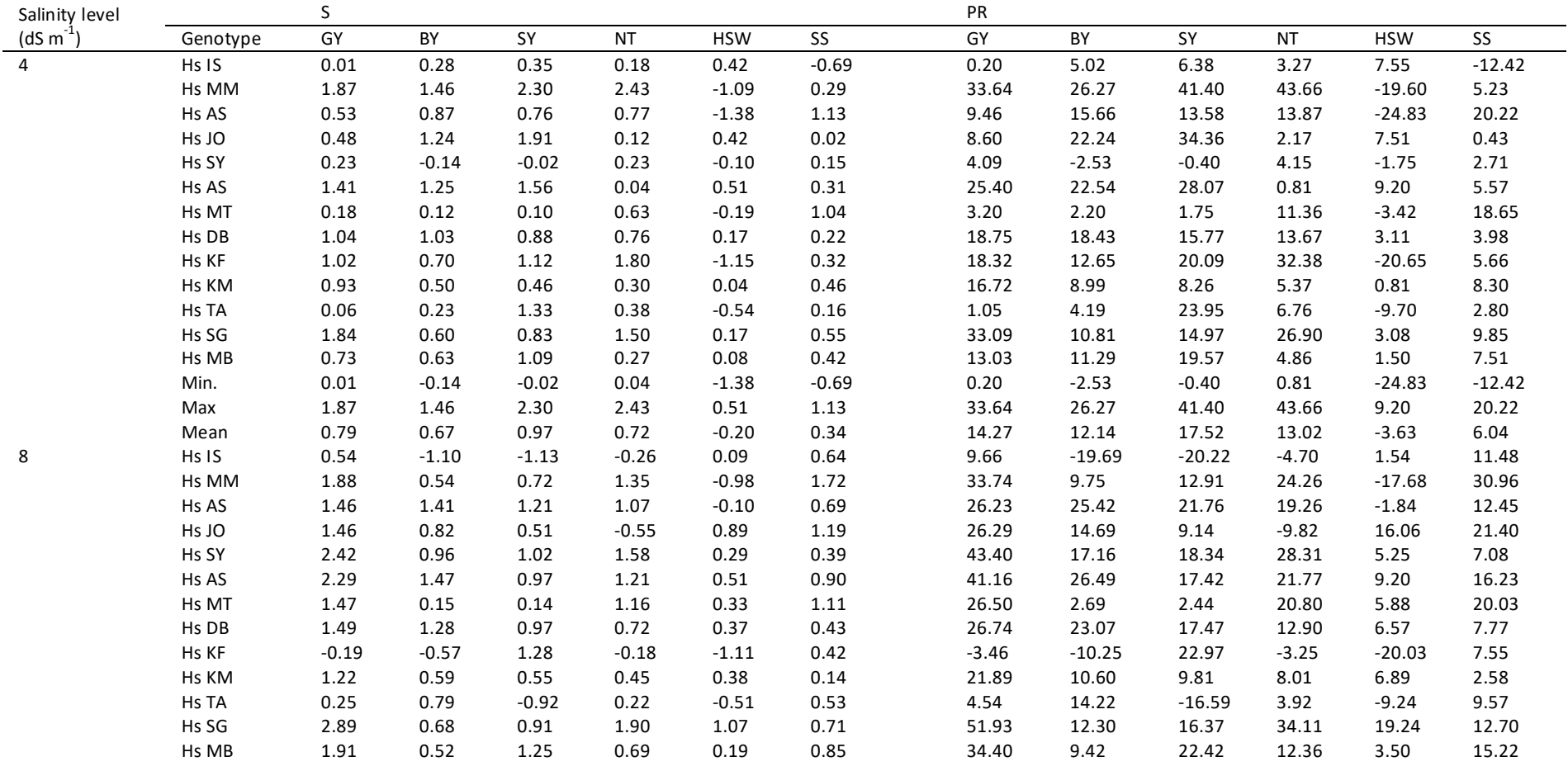




\begin{tabular}{|c|c|c|c|c|c|c|c|c|c|c|c|c|c|}
\hline & Min. & -0.19 & -1.10 & -1.13 & -0.55 & -1.11 & 0.14 & -3.46 & -19.69 & -20.22 & -9.82 & -20.03 & 2.58 \\
\hline & Max & 3.64 & 1.47 & 1.28 & 2.30 & 1.26 & 1.72 & 65.33 & 26.49 & 22.97 & 41.31 & 22.73 & 30.96 \\
\hline & Mean & 1.57 & 0.50 & 0.49 & 0.83 & 0.17 & 0.76 & 28.17 & 8.96 & 8.85 & 14.82 & 2.96 & 13.65 \\
\hline \multirow[t]{16}{*}{12} & Hs IS & 3.64 & -0.76 & -1.05 & 2.30 & 1.26 & 1.32 & 65.33 & -13.66 & -18.94 & 41.31 & 22.73 & 23.74 \\
\hline & Hs MM & 3.04 & -0.03 & -0.18 & 1.69 & 0.65 & 1.50 & 54.64 & -0.55 & -3.19 & 30.29 & 11.61 & 26.95 \\
\hline & Hs AS & 1.68 & 1.03 & 0.61 & 1.19 & -0.16 & 0.77 & 30.18 & 18.56 & 10.97 & 21.32 & -2.79 & 13.79 \\
\hline & Hs JO & 2.35 & 0.66 & 0.28 & 1.19 & 0.94 & 0.81 & 42.16 & 11.89 & 5.05 & 21.41 & 16.84 & 14.56 \\
\hline & Hs SY & 2.00 & 1.01 & 1.06 & 1.62 & 0.01 & 0.32 & 35.94 & 18.11 & 19.10 & 29.12 & 0.14 & 5.77 \\
\hline & Hs AS & 2.69 & 1.35 & 0.80 & 1.68 & 0.39 & 1.02 & 48.25 & 24.31 & 14.46 & 30.12 & 7.10 & 18.36 \\
\hline & Hs MT & 2.07 & 0.60 & 0.38 & 1.38 & 0.31 & 1.42 & 37.15 & 10.85 & 6.85 & 24.77 & 5.56 & 25.50 \\
\hline & Hs DB & 1.84 & 1.07 & 1.04 & 1.14 & 0.57 & 0.46 & 33.10 & 19.31 & 18.76 & 20.44 & 10.17 & 8.33 \\
\hline & $\mathrm{Hs} \mathrm{KF}$ & 0.65 & 0.19 & 1.12 & 0.75 & -0.39 & 0.66 & 11.62 & 3.41 & 20.17 & 13.54 & -7.09 & 11.94 \\
\hline & Hs KM & 1.60 & 0.85 & 0.81 & 1.04 & 0.43 & 0.26 & 28.71 & 15.26 & 14.59 & 18.65 & 7.77 & 4.65 \\
\hline & Hs TA & 0.32 & -0.75 & -0.49 & -0.45 & -0.06 & 0.83 & 5.67 & -13.51 & -8.88 & -8.11 & -0.99 & 14.95 \\
\hline & Hs SG & 3.04 & 1.26 & 1.29 & 1.97 & 1.26 & 0.93 & 54.69 & 22.62 & 23.13 & 35.45 & 22.71 & 16.70 \\
\hline & Hs MB & 2.13 & 0.62 & 1.27 & 0.96 & 0.74 & 0.96 & 38.24 & 11.18 & 22.76 & 17.27 & 13.21 & 17.31 \\
\hline & Min. & 0.32 & -1.11 & -1.43 & -0.45 & -0.39 & 0.26 & 5.67 & -19.93 & -25.70 & -8.11 & -7.09 & 4.65 \\
\hline & Max & 3.64 & 1.47 & 1.29 & 2.30 & 1.28 & 1.72 & 65.33 & 26.49 & 23.13 & 41.31 & 23.06 & 30.96 \\
\hline & Mean & 2.14 & 0.58 & 0.56 & 1.26 & 0.49 & 0.94 & 38.40 & 10.46 & 9.99 & 22.68 & 8.87 & 16.88 \\
\hline \multirow[t]{16}{*}{16} & Hs IS & 3.46 & -1.11 & -1.43 & 1.66 & 1.28 & 1.66 & 62.18 & -19.93 & -25.70 & 29.82 & 23.06 & 29.76 \\
\hline & Hs MM & 3.13 & 0.35 & 0.13 & 1.57 & 0.93 & 1.72 & 56.27 & 6.36 & 2.30 & 28.17 & 16.68 & 30.90 \\
\hline & Hs AS & 2.08 & 1.21 & 0.96 & 1.28 & 0.27 & 1.02 & 37.37 & 21.82 & 17.24 & 23.04 & 4.85 & 18.39 \\
\hline & Hs JO & 3.20 & 0.91 & 0.57 & 1.30 & 1.92 & 1.11 & 57.46 & 16.32 & 10.18 & 23.43 & 34.52 & 19.97 \\
\hline & Hs SY & 2.33 & 1.21 & 1.25 & 1.71 & -0.09 & 0.81 & 41.86 & 21.68 & 22.43 & 30.73 & -1.61 & 14.62 \\
\hline & $\mathrm{Hs}$ AS & 2.65 & 1.70 & 1.21 & 1.77 & 0.68 & 0.43 & 47.69 & 30.58 & 21.81 & 31.85 & 12.24 & 7.80 \\
\hline & Hs MT & 2.27 & 1.03 & 1.22 & 1.44 & 0.58 & 1.57 & 40.75 & 18.59 & 21.97 & 25.87 & 10.49 & 28.19 \\
\hline & $\mathrm{Hs} D \mathrm{DB}$ & 2.03 & 0.15 & 1.05 & 1.09 & 0.56 & 0.81 & 36.46 & 2.78 & 18.90 & 19.56 & 10.10 & 14.58 \\
\hline & Hs KF & 0.78 & 1.32 & 2.49 & 0.79 & -0.70 & 0.92 & 14.03 & 23.64 & 44.69 & 14.17 & -12.55 & 16.60 \\
\hline & Hs KM & 2.27 & 2.45 & 2.61 & 1.39 & 0.42 & 0.53 & 40.81 & 44.01 & 46.93 & 24.89 & 7.55 & 9.49 \\
\hline & Hs TA & 0.78 & 0.70 & 1.10 & -0.75 & 0.43 & 0.95 & 14.08 & 12.60 & 19.77 & -13.51 & 7.79 & 17.14 \\
\hline & Hs SG & 3.30 & 1.83 & 1.99 & 2.07 & 1.19 & 1.26 & 59.38 & 32.79 & 35.67 & 37.25 & 21.45 & 22.64 \\
\hline & Hs MB & 2.89 & 1.32 & 2.44 & 1.19 & 1.20 & 1.12 & 52.01 & 23.69 & 43.82 & 21.30 & 21.50 & 20.09 \\
\hline & Min. & 0.32 & -1.11 & -1.43 & -0.75 & -0.70 & 0.26 & 5.67 & -19.93 & -25.70 & -13.51 & -12.55 & 4.65 \\
\hline & Max & 3.64 & 2.45 & 2.61 & 2.30 & 1.92 & 1.72 & 65.33 & 44.01 & 46.93 & 41.31 & 34.52 & 30.96 \\
\hline & Mean & 2.25 & 1.01 & 1.16 & 1.20 & 0.58 & 1.01 & 40.50 & 18.13 & 20.88 & 21.51 & 10.52 & 18.19 \\
\hline
\end{tabular}

$\mathrm{PH}$, plant
period.

Table 6. Salt susceptibility index (S) and percent of reduction (PR) in agronomic traits of 13 barley landraces and two improved varieties of barley.

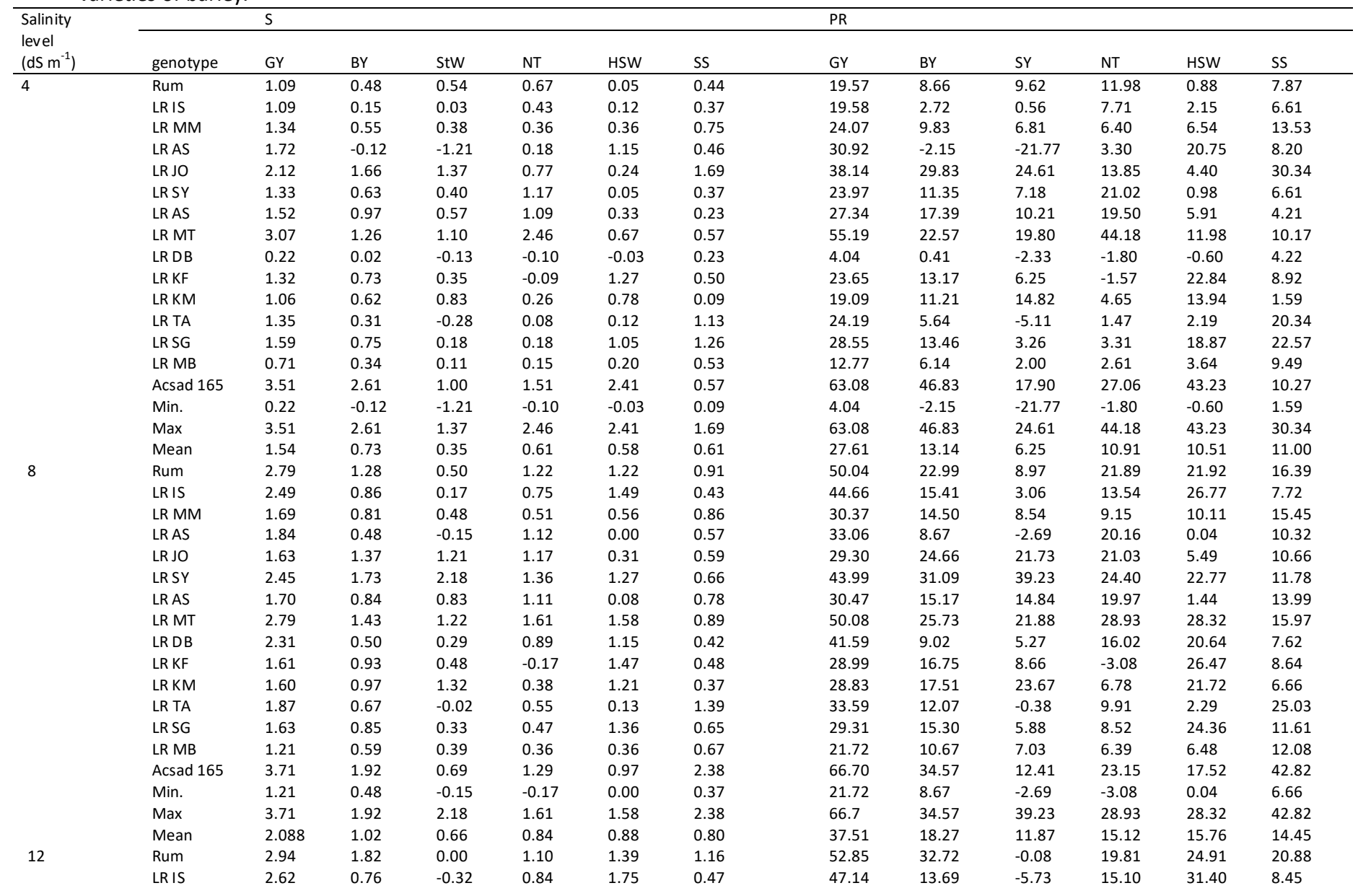




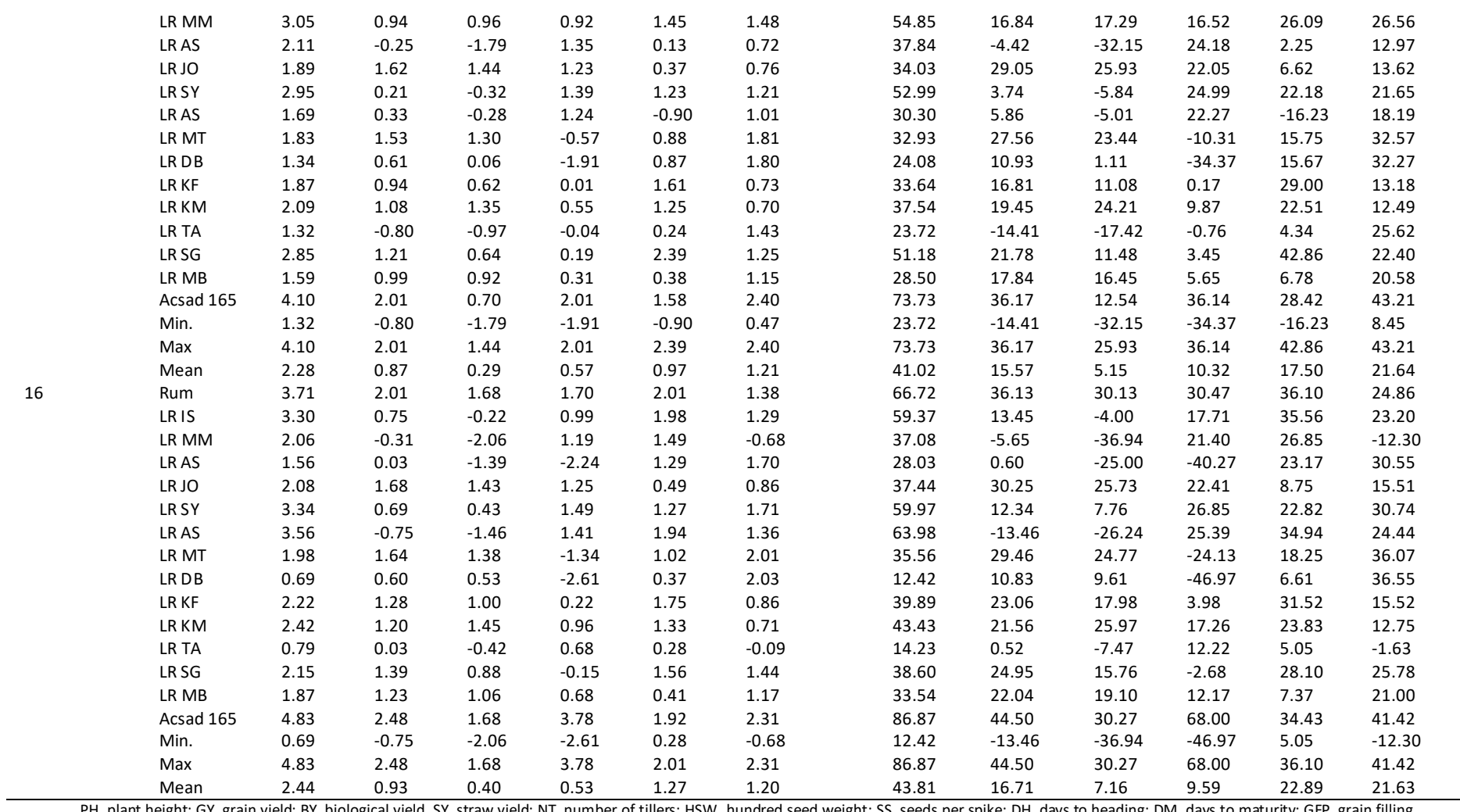
period.

Table 7. Estimates of variance components, broad sense heritability $\left(h^{2}\right)$ and $95 \%$ confidence interval of $h^{2}$ for the final germination percentage (FGP) and seedling traits.

\begin{tabular}{llllll}
\hline \multicolumn{5}{c}{ Source of variation } \\
\cline { 2 - 6 } Trait & Genotype & Genotypes $\times$ Salinity levels & Error & $h^{2}$ & C.I. $\left(h^{2}\right)$ \\
\hline FGP & $306.78^{* *}$ & $12.99^{* *}$ & 17.76 & 98.03 & $96.19-98.87$ \\
NSR & $0.48^{* *}$ & -0.05 & 0.24 & 92.58 & $85.61-95.72$ \\
SL & $3.63^{* *}$ & $0.47^{* *}$ & 3.09 & 83.60 & $68.19-90.55$ \\
CL & $0.25^{* *}$ & 0.01 & 0.13 & 89.99 & $80.59-94.23$ \\
SRL & $1.16^{* *}$ & -0.14 & 1.93 & 76.37 & $54.16-86.38$ \\
SDW & $7.16^{* *}$ & -2.34 & 6.97 & 88.54 & $77.78-93.40$ \\
RDW & $1.18^{* *}$ & 0.11 & 1.10 & 83.01 & $67.05-90.21$ \\
RDW:SDW & $0.0027^{*}$ & 0.00 & 0.015 & 47.70 & $-1.44-69.86$ \\
SRL:SL & $0.007+$ & $0.0311^{* *}$ & 0.032 & 35.95 & $-24.23-63.09$ \\
\hline
\end{tabular}

FGP, final germination percentage; NSR, number of seminal roots; SL, seedling length; CL, coleoptile length; SRL, seminal root length, SDW, shoot dry weight; RDW, root dry weight.

Table 8. Estimates of variance components, broad sense heritability $\left(h^{2}\right)$ and $95 \%$ confidence interval of $h^{2}$ for agronomic traits of genotypes.

\begin{tabular}{llllll}
\hline \multicolumn{5}{c}{ Source of variation } \\
\hline Trait & Genotype & Genotypes $\times$ Salinity levels & Error & $h^{2}$ & C.I. $\left(h^{2}\right)$ \\
\hline PH & $69.83^{* *}$ & $86.83^{* *}$ & 33.42 & 74.38 & $50.31-85.24$ \\
GY & $0.14^{* *}$ & -0.0072 & 0.1104 & 86.92 & 74.6492 .47 \\
BY & $0.27^{* *}$ & -0.06 & 0.64 & 70.03 & $41.86-82.73$ \\
SY & $0.26^{* *}$ & -0.0089 & 0.34 & 79.94 & 61.1088 .44 \\
HI & $0.0028^{* *}$ & $0.0011^{* *}$ & 0.0016 & 83.88 & 68.7390 .71 \\
NT & $0.52^{* *}$ & $0.08+$ & 0.36 & 85.54 & 71.9491 .66 \\
HSW & $0.96^{* *}$ & $0.11^{* *}$ & 0.13 & 95.29 & $90.85-97.28$ \\
SS & $4.59^{* *}$ & -0.99 & 1.91 & 96.14 & 92.5297 .78 \\
DH & $4.51^{* *}$ & $18.50^{* *}$ & 5.93 & 48.03 & $-0.80-70.05$ \\
DM & $13.30^{* *}$ & $14.55^{* *}$ & 3.10 & 79.02 & $59.31-87.91$ \\
GFP & 0.13 & $12.60^{* *}$ & 8.57 & 3.07 & $-88.02-44.14$ \\
Proline content & $9.41^{* *}$ & $10.61^{* *}$ & 9.41 & 69.53 & $40.89-82.44$ \\
\hline
\end{tabular}

*,** Significant at the 0.05 and 0.01 probability level, respectively.
PH, plant height; GY, grain yield; BY, biological yield; SY, straw yield; HI, harvest index; NT, number of tillers; HSW, hundred seed weight; $\mathrm{SS}$, seeds per spike; DH, days to heading; DM, days to maturity; GFP, grain filling period. 
drought stress tolerance. The results of this study indicate that a substantial amount of variation existed in the assessed barley materials. The response pattern of genotypes to salt stress was significantly different, which also indicates that selection for tolerance within the germplasm is possible to improve barley performance in salt-prone regions. Landraces and wild genotypes used in this study can be desirable sources of genes for the improvement of the barley crop for salt stress tolerance. In this study, there was a consistent high genetic variance component for most of assessed traits, which indicates a strong genetic potential overcoming salt tolerance. This result is supported by previous studies (Yeo and Flowers, 1986; Kumar and Narayana, 2013; Long et al., 2013; Kumar et al., 2014; Sbei et al., 2015). The relatively high $h^{2}$ estimates (0.48-0.98) for most assessed traits at seedling and reproduction stages of barley indicate a high potential for salt tolerance. This finding is in agreement with previous studies (Ashraf et al., 1986; Vysotskaya et al., 2010; Liatukas and Ruzgas, 2011; Long et al., 2013), which may lead to high expected genetic gains from direct or indirect selection in plant breeding programs. The positive correlation amongst number of seminal roots, the lengths of seedling, coleoptile and seminal root and seedling dry weight, indicating that selection for seedling related-traits seems to be good selection criteria for a vigorous seedling under salt stress. The results of this study have shown that yield-contributing traits such as number of tillers plant ${ }^{-1}$, number of kernels spike $^{-1}$ and hundred-kernel weight were genetically controlled and were correlated with yield. However, grain yield plant ${ }^{-1}$ was positively but weakly associated with the number of tillers plant ${ }^{-1}$ and number of seeds spike ${ }^{-1}$ and showed a strong positive genetic correlation with hundredkernel weight. As a result, kernel weight can be considered as an indirect selection criterion to improve grain yield under salt stress.

In this study, all genotypes showed a high germination percentage, therefore, they would be desirable over other genotypes that are significantly incapable of being recovered to germinate under salt stress. This result is in line with previous studies (Mer et al., 2000; Kaya et al., 2006; Ibrahim, 2016) that indicated the adverse effect of salt stress on germination. The reduction in germination percentage under salt stress can be attributed to the high osmotic potential and the toxic effect of some ions in the growing medium such as $\mathrm{Na}, \mathrm{Ca}, \mathrm{Mg}, \mathrm{Cl}$ and $\mathrm{SO}_{4}$ ions (Huang and Redmann, 1995; Tavakkoli et al., 2011; Flowers et al., 2015).

Seedling traits were adversely decreased by increasing the level of salinity. However, the reduction in seminal root growth was greater than the reduction in shoot length. The high sensitivity of root to salt stress, likely because the direct exposure of roots to osmotic stress and the specific toxicity of ions in the growing medium (Jaradat et al., 2004b). Direct selection for grain yield and indirect selection for yield contributing traits seem to be a reliable trait for high yielding of barley genotypes under salt stress (Isla et al., 1998). In this study, all yield components significantly contributed in yield losses by increasing the level of salinity. The reduction in yield and yield contributing traits under salt stress are mainly attributed to low nutrients and water uptake under high salt osmotic stress and possibly the $\mathrm{Na}^{+}$ and $\mathrm{Cl}^{-}$ion toxicity (Yeo and Flowers, 1986). The considerable interaction a genotype $\times$ salt treatment on grain yield and yield contributing traits indicate the variable response of barley genotypes to salt stress. The exposure of barley genotypes to salt stress inhibited plant height, which can be attributed to the toxic effect of $\mathrm{Na}^{+}$and $\mathrm{Cl}^{-}$in the growing plant parts (Yeo and Flowers, 1986).

Accumulation of proline in the fresh leaves of barley that had exposed to salt stress, acts as an endogenous osmotic regulator. Accumulation of proline in barley tissues correlates with the ability of barley adaptation to salt stress (Stewart and Lee, 1974). This result was previously reported in barley (Pesci and Beffagana, 1986) and in other agricultural crops such as rice (Dubey and Rani, 1989) and Brassica juncea (Jain et al., 1991).

The susceptibility index ' $\mathrm{S}$ ' (Bruckner and Frohberg, 1987) reflects the relative yield loss $\left(1-Y_{D} / Y_{P}\right)$ of an individual genotype relative to the mean yield losses of all genotypes tested in the experiment $\left(1-X_{D} / X_{P}\right)$. This index could be used to rank genotypes according to their relative salt tolerance; such index takes into account the relative yield loss but not the yield potential of the genotypes. The low $\mathrm{S}$ value for a certain genotype as compared with other genotypes indicates that the lower yield loss under salt stress as compared with no stress conditions and contrariwise. The low $S$ value is an indication for salt tolerance, for example, genotypes LR DB ( $S=0.69)$, Hs TA (S $=0.78)$ and $\operatorname{LR} T A(S=0.79)$ were the most tolerant genotypes. The ranking response pattern of salt tolerance of Hs TA and LR TA based on grain yield was consistent with the ranking response pattern based on germination and seedling related-traits. However, other genotypes showed no association in salt tolerance at the first stages of the life cycle and maturity. The highest reduction in grain yield was detected in the two improved varieties, implying higher susceptibility to salt stress. Tolerance to external environmental stresses depends on the stage of plant development, where the level of tolerance does not necessarily correlate with the level of tolerance at other stages of plant development (Foolad et al., 1998; Long et al., 2013). In addition, Isla et al. (1998) reported the incapability to identify a combination of traits that is useful as selection criteria in barley under salt stress. However, there is great possibility to identify genotypes that are tolerant to drought at different growth stages, for example, El-Hendawy et al. (2005) assessed the tolerance of thirteen wheat genotypes under salt stress and some genotypes displayed potential to tolerate salt stress at different stages of growth development. Consequently, direct selection for grain yield is very reliable to evaluate the genotypes with a high potential yield under salt stress.

\section{Materials and methods}

\section{Plant materials}

A total of (26) barley population (i.e. accessions) from barley landrace and its wild progenitor $(H$. spontaneum) were collected from 13 different sites (i.e. one landrace and one wild barley sample from each site) in Jordan extending from Irbid in the north to Ma'an in the south as shown in Fig 1. Each sample was collected from 30 different spikes at least 1 meter apart. Locations at lower altitudes were visited first because of flowering and ripening had started earlier in the spring. The collection of twenty-six genotypes, in addition to two six-rowed local varieties (Rum and Acsad 176) were included in as checks and evaluated for salinity stress tolerance at the germination and the early seedling growth stages. 


\section{Experimental design}

The laboratory study was arranged in a factorial completely randomized design with one dish per replicate and five replicates per treatment (genotype and salt stress level). The pot study was arranged in a factorial completely randomized design with one pot per replicate that contains three plants and four replicates per treatment.

\section{Conduction of study}

\section{Laboratory experiment}

Each of 20 uniform size seeds was sterilized for 10 minutes in $0.5 \% \mathrm{NaOCl}$ solution and rinsed thoroughly in sterile distilled water. Seeds were placed into Petri dishes $(100 \mathrm{~mm}$ in diameter) containing two Whatman filter paper. Petri dishes were moistened with tap water of $0.85 \mathrm{dS} \mathrm{m}^{-1}$ and one of the following saline solutions of $4,8,12$ or $16 \mathrm{dS} \mathrm{m}^{-1}$ comprised of $\mathrm{NaCl}, \mathrm{CaCl}_{2}$ and $\mathrm{MgSO}_{4}$ (1:1:1 ratio). Plates were tightly sealed with parafilm $\left(\mathrm{O}_{2}\right.$ permeable) to prevent evaporation of water and minimize changes in the concentration of solutions. Plates were incubated upside down at $20 / 16^{\circ} \mathrm{C}$ day/night cycle and 16 -h photoperiod under cool white fluorescent light. Germination was observed on $24 \mathrm{~h}$ intervals for 14 days. Seeds were considered germinated if the radical was at least $2 \mathrm{~mm}$ in length. Thereafter, seedling and seminal root lengths were evaluated from five seedlings per plate.

\section{Pot experiment}

Seeds were sown in $20 \mathrm{~cm}$-diameter and $40 \mathrm{~cm}$ deep plastic pots filled with $8 \mathrm{~kg}$ of air-dried soil that sieved through a 2 $\mathrm{mm}$ mesh. The seedlings were maintained in a greenhouse at about $20 / 16^{\circ} \mathrm{C}$ (day/night) temperature, with a $16-\mathrm{h}$ photoperiod and 200-250 $\mu \mathrm{E}$ m-2 s-1 light intensity from high-pressure sodium and incandescent bulbs, and watered as needed. The plants were irrigated with half-strength Hogland solution for 10 days before starting salinization. The plants in each pot were irrigated until the soil reached $85 \%$ from its field capacity. Saline solutions were prepared, and treatments started 10 days after sowing of barley grains. Irrigation with saline solution was continued on every other two days with tap water of $0.85 \mathrm{dS} \mathrm{m}^{-1}$, or with the other saline solutions of $4,8,12$ or $16 \mathrm{dS} \mathrm{m}^{-1}$ until maturity. Barley plants exposed to different treatments of salinity were assessed using the phenological growth, and yield parameters. The phenological traits included days to heading; the time required in days from sowing until $50 \%$ of spikes fully emerged from flag leaf and days to maturity; the time required from sowing until $50 \%$ of peduncles turned yellow. The growth parameters included plant height at maturity, measured in $\mathrm{cm}$ from soil surface to the tip of spike excluding awns, grain yield per plant, number of fertile tillers per plant and 1000-grain weight (g). Grain filling period (days) was calculated by subtracting the value obtained for days to heading from the value obtained for days to maturity. Number of seeds per spike was calculated by dividing the total number of grains per plant by number of fertile tillers per plant. The proline accumulation was determined according to the standard procedure used by Bates et al. (1973).

\section{Statistical analysis}

Data from each experiment were analyzed separately using the statistical analysis system (SAS). Analysis of variance (ANOVA) was conducted using PROC MIXED to test genotype and salt as well as their interactive effects. Differences are significant at $P=0.05$ unless otherwise stated. Salinity stress susceptibility index (S) was calculated following the standard method used by Bruchner and Frohberg (1987) using the following formula:

$$
S=\frac{1-Y D / Y P}{D}
$$

Where, $Y D=$ the mean of a particular trait in salt treatment, $Y P=$ mean value under non-stress treatment and $D=1$ (mean YD of all genotypes under salt stress/mean YP of all genotypes under non-stress treatment). ' $S$ ' represents the relative susceptibility of a genotype to salt stress and ' $D$ ' represents the relative stress intensity of a treatment. For each genotype, 'S' values at the four different salt levels were estimated and then averaged to calculate the mean salinity susceptibility index for each genotype over all salinity treatments.

Growth reduction resulted from salinity stress for each trait was calculated according to the following formula:

$$
G I(\%)=\frac{(W l-S l)}{W I} * 100 \%
$$

Where, $\mathrm{WI}$ and $\mathrm{SI}$ are the value of recorded traits under salt stress and non-salt stress treatments, respectively. Selection of traits and the heritability estimates were assessed to predict the potential genetic gain. Combined analysis of variance across treatments was performed and variance components were estimated according to Snedecor and Cochran (1980). The effects of environments were fixed factors, and all other effects were random variables. Broad sense heritability on plot basis was calculated according to Knapp and Briges (1987). All statistical analyses were performed using the computer program PLABSTAT (Utz, 2000).

\section{Conclusion}

A high level of variability of barley landrace and $H$. spontaneum in response to salt tolerance from Jordan was evaluated at different stages of plant development including germination, seedling, and maturity stages. The findings of the present study indicates that selection for tolerance within the germplasm is possible to improve barley performance in salt-prone regions. Landrace and $H$. spontaneum genotypes used in this study can be desirable sources of genes for the improvement of the barley crop for salt stress tolerance. In addition, some barley landraces and $H$. spontaneum genotypes were detected to be tolerant to salt stress, and therefore, these genotypes are potentially suitable for breeding to tolerate salt stress. The relatively high $h^{2}$ estimates $(0.48-0.95)$ for most assessed traits at seedling and maturity stages of barley indicates a high potential for salt tolerance. Based on this finding, it can be concluded that a high genetic gain is expected from direct or indirect selection in plant breeding programs. Molecular analysis can be used to detect polymorphisms within a 
population and identify DNA markers associated with salt tolerance using modern SSR or SNP markers. However, genetic materials that are used in this study could be included in future studies and genome wide association studies to build up barley population and identify genomic regions underlying genes controlling drought tolerance.

\section{Disclaimer statement}

We hereby declare that this manuscript contains no materials which has been accepted for the award of any degree or diploma in any university, and that, to the best of our knowledge and belief, the manuscript contains no copy of any material previously published or written by another person except where due reference is made in the text.

\section{Acknowledgment}

We thank the Deanship of Scientific Research at Mutah University for funding this research project.

\section{References}

Abdel-Ghani AH (2009) Response of wheat varieties from semi-arid regions of Jordan to salt stress. J Agron Crop Sci. 195: 55-65.

Abdel-Ghani AH, Neumann K, Wabila C, Sharma R, Dhanagond S, Owais SJ, Börner A, Graner A, Kilian B (2014) Diversity of germination and seedling traits in a spring barley (Hordeum vulgare L.) collection under drought simulated conditions. Genet Resour Crop Ev. 62(2): 275292.

Abdel-Ghani AH, Parzies HK, Omary A, Geiger HH (2004) Estimating the outcrossing rate of barley landraces and wild barley populations collected from ecologically different regions of Jordan. Theor Appl Genet. 109: 588595.

Adjel F, Kadi Z, Bouzerzour H, Benmahammed A (2013) Salt stress effects on seed germination and seedling growth of barley (Hordeum Vulgare L.) genotypes. Int J Agr Sustain. 3(2): 223-237.

Allel D, BenAmar A, Badri M, Abdelly C (2019) Evaluation of salinity tolerance indices in North African barley accessions at reproductive stage. Czech J Genet Plant. 55: 61-69.

Ashraf M, Mceilly T, Bradshaw AD (1986) Tolerance of sodium chloride and its genetic basis in natural populations of four grass species. New Phytol. 103: 725734.

Bates LS, Waldren RP, Teare ID (1973) Rapid determination of free proline for water-stress studies. Plant Soil. 39: 205207.

Bernstein L (1962) Salts affected soils and plants. In Proceedings of the Paris symposium, UNESCO, pp. 139174.

Blum A, Golan G, Mayer J, Sinmena B, Shpiler L, Burra L (1989) The drought response of landraces of wheat from northern Negev desert. Euphytica. 43: 87-96.

Bruchner PL, Frohberg RC (1987) Stress tolerance and adaptation in spring wheat. Crop Sci. 27, 31-36.

Carillo P, Grazia M, Pontecorvo G, Fuggi A, Woodrow P (2012) Salinity stress and salt tolerance. In abiotic stress in plants - mechanisms and adaptations.

Chen Z, Zhou M, Newman IA, Mendham NJ, Zhang G, Shabala S (2007) Potassium and sodium relations in salinized barley tissues as a basis of differential salt tolerance. Funct Plant Biol. 145(4): 1714-1725.

Deinlein U, Stephan AB, Horie T, Luo W, Xu G, Schroeder J (2014) Plant salt-tolerance mechanisms. Trends Plant Sci. 19(6): 371-9.

Dubey RS, Rani M (1989) Influence of $\mathrm{NaCl}$ salinity on growth and metabolic status of protein and amino acids in rice seedlings. J Agron Crop Sci. 162: 97-106.

El-Hendawy SE, Hu Y, Yakout GM, Awad AM, Hafiz SE, Schmidhalter U (2005) Evaluating salt tolerance of wheat genotypes using multiple parameters. Eur J Agron. 22: 243-253.

Flowers TJ, Hajibagheri MA (2001) Salinity tolerance in Hordeum vulgare: Ion concentrations in root cells of cultivars differing in salt tolerance. Plant Soil. 231: 1-9.

Flowers TJ, Munns R, Colmer TD (2015) Sodium chloride toxicity and the cellular basis of salt tolerance in halophytes. Ann Bot-London 115(3): 419-431.

Foolad MR, Chen FQ, Lin GY (1998) RFLP mapping of QTLs conferring salt tolerance during germination in an interspecific cross of tomato. Theor Appl Genet. 97: 11331144.

Forster B, Law C, Nevo E, Powell W (1990) Genetic strategies for improving the salt tolerance in wheat and barley. In Proceeding of the International Symposium on Molecular and Genetic approaches to Plant Stress. New Delhi, India.

Frankel H, Burdon J, Peacock J (1995) Landraces in transit The threat perceived. Div. 11: 14-15.

Gupta B, Huang B (2014) Mechanism of salinity tolerance in plants: Physiological, biochemical, and molecular characterization. Int J Genomics 2014(1), Article ID: 701596.

Harlan J (1975) Our vanishing genetic resources. Science. 188: 618-621.

Harlan JR (1976) Evolution of Crop Plants. In: Simmonds NW (ed), Longman, London.

Huang J, Redmann RE (1995) Salt tolerance of Hordeum and Brassica species during germination and early seedling growth. Can J Plant Sci. 75: 815-819.

Ibrahim EA (2016) Seed priming to alleviate salinity stress in germinating seeds. J Plant Physiol. 192: 38-46.

Isla R, Aragüés R, Royo A (1998) Validity of various physiological traits as screening criteria for salt tolerance in barley. Field Crop Res. 58: 97-107.

Jain S, Nainawatee HS, Jain RK, Chowdhury JB (1991) Proline status of genetically stable salt-tolerant Brassica juncea L. somaclones and their parent cv. Prakash. Plant Cell Rep. 9: 684-687.

Jaradat AA (1991) Grain protein variability among populations of wild barley (Hordeum spontaneum C-Koch) from Jordan. Theor Appl Genet. 83: 164-168.

Jaradat AA, Shahid M, Al-Maskri A (2004a) Genetic diversity in the Batini barley landrace from Oman: I. spike and seed quantitative and qualitative traits. Crop Sci. 44: 997-1007.

Jaradat AA, Shahid M, Al-Maskri A (2004b) Genetic diversity in the Batini barley landrace from Oman: II Response to salinity stress. Crop Sci. 44: 997-1007.

Kaya MD, Okçu G, Atak M, Çikili Y, Kolsarici Ö (2006) Seed treatments to overcome salt and drought stress during germination in sunflower (Helianthus annuus L.). Eur J Agron. 24: 291-295.

Knapp SJ, Briges WL (1987) Confidence interval estimates for heritability for several mating and experimental designs. Theor Appl Genet. 73: 759-763. 
Koornneef M, Alonso-Blanco C, Peters AJM (1997) Genetic approaches in plant physiology. New Phytol. 137: 1-8.

Kramer PJ, Boyer JS (1996) Water relations of plants and soils. Soil Sci. 161: 42-79.

Kumar B, Abdel-Ghani AH, Pace J, Reyes-Matamoros J, Hochholdinger F, Lübberstedt T (2014) Association analysis of single nucleotide polymorphisms in candidate genes with root traits in maize (Zea mays L.) seedlings. Plant Sci. 224: 9-19.

Kumar B, Narayana T (2013) Candidate gene based association study for nitrogen use efficiency and associated traits in maize. Doctor of Philosophy Dissertation.

Liatukas Ž, Ruzgas V (2011) Relationship of coleoptile length and plant height in winter wheat accessions. Pakistan J Bot. 43: 1535-1540.

Long NV, Dolstra O, Malosetti M, Kilian B, Graner A, Visser RGF, van der Linden CG (2013) Association mapping of salt tolerance in barley (Hordeum vulgare L.). Theor Appl Genet. 126(9): 2335-2351.

Maas E (1986) Salt tolerance of plants. Appl Agri Res. 1: 1226.

Mer RK, Prajith PK, Pandya DH, Pandey AN (2000) Effect of salts on germination of seeds and growth of young plants of Hordeum vulgare, Triticum aestivum, Cicer arietinum and Brassica juncea. J Agron Crop Sci. 185(4): 209-217.

Morrell PL, Clegg MT (2007) Genetic evidence for a second domestication of barley (Hordeum vulgare) east of the Fertile Crescent. Proceedings of the National Academy of Sciences.

Nevo E, Beiles A, Gutterman Y, Storch N, Kaplan D (1984) Genetic resources of wild cereals in Israel and vicinity II. Phenotypic variation within and between populations of wild barley (Hordeum sponataneum). Euphytica. 33: 737756.

Nevo E, Chen G (2010) Drought and salt tolerances in wild relatives for wheat and barley improvement. Plant Cell Environ. 33(4): 670-685.

Okçu G, Kaya MD, Atak M (2005) Effects of salt and drought stresses on germination and seedling growth of pea (Pisum sativum L.). Turk J Agric For. 29: 237-242.

Omara M, Hussein M (1987) Selection for salt tolerance in barley. Assiut J Agric Sci. 18: 199-218.

Parida AK, Das AB (2005) Salt tolerance and salinity effects on plants: a review. Ecotox Environ safe. 60(3): 324-349.

Pesci P, Beffagna N (1986) Influence of exogenously supplied potassium and sodium salts on the abscisic acid-induced proline accumulation in barley leaf segments. Physiol Plant. 67: 123-128.

Rahnama A, Munns R, Poustini K, Watt M (2011) A screening method to identify genetic variation in root growth response to a salinity gradient. J Exp Bot. 62: 69-77.

Roy SJ, Negrão S, Tester M (2014) Salt resistant crop plants. Current opinion in biotechnology.
Sbei H, Sato K, Shehzad T, Harrabi M, Okuno K (2015) Detection of QTLS for salt tolerance in Asian barley (Hordeum vulgare L.) by association analysis with SNP markers. Breed Sci. 64(4): 378-388.

Shabala S, Chen ZH, Zhou MX, Newman IA, Mendham NJ, Zhang GP (2007) Potassium and sodium relations in salinized barley tissues as a basis of differential salt tolerance. Funct Plant Biol. 34: 150-162.

Sinha SK (1987) Drought resistance in crop plants: A critical physiological and biochemical assessment. In: Srivastava JP, Porceddu E, Acevedo E, Varma S (eds.) Drought Tolerance in Winter Cereals. John Wiley and Sons, England, pp 349-364.

Snedecor GW, Cochran WG (1980) Statistical methods, 7th edition. lowa State Univ. Press, Ames, IA.

Sousa WP, Kennedy PG, Mitchell BJ (2003) Propagule size and predispersal damage by insects affect establishment and early growth of mangrove seedlings. Oecologia. 135: 564-575.

Stewart GR, Lee JA (1974) The role of proline accumulation in halophytes. Planta. 120: 279-289.

Takahashi H, Sato K, Takeda K (2001) Mapping genes for deep-seeding tolerance in barley. Euphytica. 122: 37-43.

Tavakkoli E, Fatehi F, Coventry S, Rengasamy P, McDonald GK (2011) Additive effects of $\mathrm{Na}+$ and $\mathrm{Cl}$ - ions on barley growth under salinity stress. J Exp Bot. 62(6): 2189-203.

Troech FR, Thompson LM (1993) Soils and soil fertility. Oxford University Press, New York.

Utz H (2000) PLABSTAT. A computer program for statistical analysis of plant breeding experiments. Second version. Institute of Plant Breeding, Seed Science and Population Genetics, University of Hohenheim, Stuttgart, Germany.

Vysotskaya L, Hedley PE, Sharipova G, Veselov D, Kudoyarova G, Morris J, Jones HG (2010) Effect of salinity on water relations of wild barley plants differing in salt tolerance. AoB Plants. plq006.

Weltzien E (1989) Differentiation among barley landrace populations from the Near East. Euphytica. 43: 29-39.

Weltzien E, Fischbeck G (1990) Performance and variability of local barley landraces in near-eastern environments. Plant Breed. 104: 58-67.

Yeo AR, Flowers TJ (1986) Salinity resistance in rice (Oryza sativa $\mathrm{L}$.) and a pyramiding approach to breeding varieties for saline soils. Funct Plant Biol. 13: 161-173.

Zhang $\mathrm{H}$, Irving LJ, McGill C, Matthew C, Zhou D, Kemp P (2010) The effects of salinity and osmotic stress on barley germination rate: Sodium as an osmotic regulator. Ann Bot-London 106(6): 1027-1035.

Zhu JK (2001) Plant salt tolerance. Trends Plant Sci. 6(2): 6671.

Zohary D, Hopf M (2000) Domestication of Plants in the Old World. Third edition University Press, Oxford 531: 206. 